\title{
Research Paper \\ Comparing the Effects of Eight Weeks of Whole Body Vibration Exercise Com- bined With Rope Skipping at Two Different Intensities on Physical Performance of Older Men: A Randomized Single-Blind Clinical Trial
}

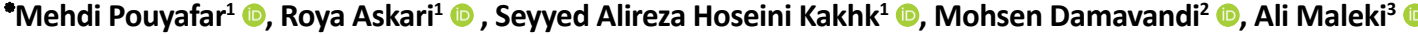

1. Department of Sports Physiology, Faculty of Sports Science, Hakim Sabzevari University University, Sabzevar, Iran. 2. Department of Sport Biomechanics, Faculty of Sports Science, Hakim Sabzevari University University, Sabzevar, Iran. 3. Department of Biomedical Engineering, Science Campus and New Technologies, Semnan University, Semnan, Iran.

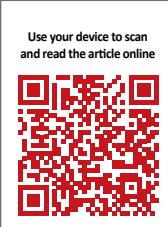

Citration: Pouyafar M, Askari R, Hosseinai Kakhk SA, Damavand M, Maleki A. [Comparing the Effects of Eight Weeks of Whole Body Vibration Exercise Combined With Rope Skipping at Two Different Intensities on Physical Performance of Older Men: A Randomized Single-Blind Clinical Trial (Persian)]. Iranian Journal of Ageing. 2021; 16(3):376-395. http://dx.doi. org/10.32598/sija.2021.16.3.2885.2

doi) $h$ http://dx.doi.org/10.32598/sija.2021.16.3.2885.2

Keywords:

Combined training Intensity, Physical performance, Elderly

\section{A B S TRACT}

Objectives Whole-Body Vibration (WBV) exercise seems to be an effective alternative to improve physical performance in the elderly. This study aims to compare the effects of eight weeks of WBV exercise combined with rope skipping at two different intensities on physical performance of older men.

Methods \& Materials This is a randomized single-blind clinical trial. Participants were 30 older men (Mean $\pm S D$ age $=65.83 \pm 4.16$ years; Mean $\pm S D$ height $=169.26 \pm 3.90 \mathrm{~cm}$; Mean $\pm S D$ weight $=77.04 \pm 4.62 \mathrm{~kg}$; Body Mass Index $=26.86 \pm 0.69 \mathrm{~kg} / \mathrm{m}^{2}$ ) living in Mashhad, Iran in 2019, who were selected by purposive and convenience sampling methods and randomly divided into three groups: high intensity $(H I ; n=12)$, low intensity $(\mathrm{LI} ; \mathrm{n}=12)$ and control $(\mathrm{n}=10)$. The WBV exercise was performed at a frequency of 25-40 $\mathrm{Hz}$ and amplitude of $3 \mathrm{~mm}$. Rope skipping was performed based on the Borg scale at 13-14 level intensities with 30-35 jumps per minute. Physical performance indicators, body mass index, and fat percentage were measured before and after eight weeks of intervention. Repeated measures analysis of variance was used for data analysis in SPSS v. 20 software. The significance level was set at $P \leq 0.05$.

Results In $\mathrm{HI}$ and LI groups, body fat percentage (-4.50\%, -5.99\%), lower body flexibility (105\%, 102\%), upper limb muscle strength (42.43\%, 42.93\%), handgrip strength (43.38\%, 39.45\%), dynamic leg strength (36.47\%, $26.43 \%)$, lower limb muscular endurance (27.20\%, 26.10\%), cardiorespiratory function (10.27\%, 10.90\%), and dynamic balance $(-32.60 \%,-24.10 \%)$ showed a significant improvement compared to the control group $(P<0.05)$. There was no significant difference in body mass index between exercise and control groups $(P>0.05)$, and no significant difference between the two exercise groups in any of the study variables $(P>0.05)$

Conclusion It seems that low intensities of the WBV exercise + rope skipping is effective in improving body composition and physical performance in older men. The selection of intensity level depends on the physical condition of the elderly.

\section{* Corresponding Author: \\ Mehdi Pouyafar, PhD.}

Address: Department of Sports Physiology, Faculty of Sports Science, Hakim Sabzevari University University, Sabzevar, Iran.

Tel: +98 (51) 44012763

E-mail: r.askari@hsu.ac.ir 


\section{Extended Abstract}

\section{Introduction}

A

ging is an unavoidable stage of human life that is associated with a decrease in strength and the amount of physical activity and mobility. With aging, physical dysfunction especially movement limitation increases. This leads to dependence on others in daily tasks. These factors can have many negative effects on the quality of life of the elderly [1]. Therefore, the importance of muscle mass and strength, especially maximal muscular strength, has a significant effect on how older people perform daily activities [2]. One of the interventions for these people is While-Body Vibration (WBV) exercises which are for people who are less inclined to participate in sports classes or people who have difficulty walking. It can also increase skeletal muscle strength in both young and old people, similar to resistance training [7].

\section{Methods}

This is a quasi-experimental study, a clinical trial $($ Code $=$ IRCT20200109046063N1) with an ethical approval from Hakim Sabzevari University (Code: IR.HSU. REC.1398.002). Participants were 34 older men (Mean $\pm \mathrm{SD}$ age $=65.83 \pm 4.16$ years; height $=169.26 \pm 3.90 \mathrm{~cm}$; weight $=77.04 \pm 4.62 \mathrm{~kg}$; Body Mass Index $=26.86 \pm 0.69 \mathrm{~kg} /$ $\mathrm{m} 2)$. They were randomly divided into three groups: high intensity or $\mathrm{HI}(\mathrm{N}=12)$, low intensity or LI $(\mathrm{N}=12)$, and control $(\mathrm{N}=10)$. Physical function tests including the tests of handgrip strength, upper limb muscle strength, dynamic leg strength, lower limb muscular endurance, dynamic balance, lower body flexibility, cardiorespiratory function, body composition, and body mass index were conducted at baseline and at the end of eight weeks of intervention. After becoming familiar with WBV machine and rope training, the two exercise groups performed WBV exercise program for 8 weeks, 3 sessions per week each 30 minutes (4 oneminute sets in the first four weeks and 5 one-minute sets in the second four weeks). The amplitude was equal to 3 millimeters for both groups. The frequency was $40 \mathrm{~Hz}$ in the first group (HI) and $25 \mathrm{~Hz}$ in the second group (LI). Rest between sets was 30-45 seconds and between movements was 90-120 seconds with specific positions of upper body (push-up position, and reverse wrist flexion) and lower body (squat and lunge positions) on the WBV machine. The control group performed their routine activities without any intervention. The rope training program combined with WBV exercises started with two sets of one minute increased to six sets of one minute in the last sessions, where there were a 30-s rest between sets and 30-35 jumps per minute. The rope intensity was measured and determined at 13 and14 levels by the Borg Scale of 6-20. Data analysis was performed in SPSS v. 20 software.

\section{Results}

The results of physical function tests and body composition are presented in Table 1. In HI and LI groups, body fat percentage $(-4.50 \%,-5.99 \%)$, lower body flexibility $(105 \%, 102 \%)$, upper limb muscle strength $(42.43 \%$, $42.93 \%)$, handgrip strength $(43.38 \%, 39.45 \%)$, dynamic leg strength $(36.47 \%, 26.43 \%)$, lower limb muscular endurance $(27.20 \%, 26.10 \%)$, Cardiorespiratory function $(10.27 \%, 10.90 \%)$, and dynamic balance $(-32.60 \%$, $-24.10 \%)$ showed a significant improvement compared to the control group $(\mathrm{P}<0.05)$. There was no significant difference in body mass index between exercise and control groups ( $\mathrm{P}>0.05)$, and no significant difference between the two exercise groups in any of the study variables $(\mathrm{P}>0.05)$, although, the HI exercise group had higher means than the LI exercise group.

\section{Discussion and Conclusion}

The WBV exercises at frequencies of 25 and $40 \mathrm{~Hz}$ and amplitude of $3 \mathrm{~mm}$ combined with rope exercises improve muscle function indicators of the elderly, but there is no significant difference between the groups that receives exercise with high and low intensities. The indicators was higher in the group received high intensity exercises which can be the recommended intensity in using the combination of WBV-rope exercises. Given that both intensity of exercises had a positive effect on muscle function indicators, older people aged 60-70 years are likely to be able to benefit from both types of exercises depending on their physical condition; however, HI exercises may provide a better level of physical fitness for them.

\section{Ethical Considerations}

\section{Compliance with ethical guidelines}

This study was approved by the ethical committee of Hakim Sabzevari University (Code: IR.HSU.REC.1398.002). All ethical principles are considered in this article. The participants were informed about the purpose of the research and its implementation stages; they were also assured about the confidentiality of their information; moreover, they were free to leave the study whenever they wished, and if desired, the research results would be available to them. 
Table 1. Mean scores of the physical function indicators

\begin{tabular}{|c|c|c|c|c|c|c|c|}
\hline \multirow{2}{*}{\multicolumn{2}{|c|}{ Variables }} & \multicolumn{3}{|c|}{ Mean $\pm S D$} & \multirow{2}{*}{ Time $^{\#}$} & \multirow{2}{*}{ Group } & \multirow{2}{*}{ Time $\times$ Group* } \\
\hline & & HI Group & LI Group & Control Group & & & \\
\hline \multirow{3}{*}{$\begin{array}{l}\text { Body Mass Index } \\
\qquad\left(\mathrm{kg} / \mathrm{m}^{2}\right)\end{array}$} & Pre-test & $26.69 \pm 0.56$ & $27.11 \pm 0.52$ & $26.78 \pm 1.00$ & & & \\
\hline & Post-test & $26.48 . \pm 0.57$ & $26.89 \pm 0.54$ & $26.74 \pm 1.02$ & 0.001 & 0.480 & 0.001 \\
\hline & Mean difference & -0.78 & -0.81 & -0.14 & & & \\
\hline \multirow{3}{*}{ Fat percentage } & Pre-test & $25.30 \pm 1.17$ & $25.53 \pm 0.90$ & $26.24 \pm 1.23$ & & & \\
\hline & Post-test & $24.16 \pm 1.14$ & $24.00 \pm 0.88$ & $26.15 \pm 1.26$ & 0.001 & 0.019 & 0.001 \\
\hline & Mean difference & -4.50 & -5.99 & -0.34 & & & \\
\hline \multirow{3}{*}{$\begin{array}{l}\text { Lower body flex- } \\
\text { ibility }(\mathrm{cm})\end{array}$} & Pre-test & $-7.89 \pm 1.96$ & $-7.89 \pm 1.83$ & $-6.62 \pm 1.99$ & & & \\
\hline & Post-test & $0.44 \pm 2.50$ & $0.22 \pm 1.78$ & $-6.75 \pm 3.24$ & 0.001 & 0.018 & 0.001 \\
\hline & Mean difference & 105 & 102 & 1.96 & & & \\
\hline \multirow{3}{*}{$\begin{array}{l}\text { Upper limb muscle } \\
\text { strength (number } \\
\text { per } 30 \text { seconds) }\end{array}$} & Pre-test & $19.11 \pm 2.61$ & $18.89 \pm 3.01$ & $19.12 \pm 1.72$ & & & \\
\hline & Post-test & $27.11 \pm 3.15$ & $27.00 \pm 3.20$ & $19.75 \pm 2.43$ & 0.001 & 0.016 & 0.001 \\
\hline & Mean difference & 42.43 & 42.93 & 3.29 & & & \\
\hline \multirow{3}{*}{$\begin{array}{l}\text { Handgrip strength } \\
\qquad(\mathrm{kg})\end{array}$} & Pre-test & $21.00 \pm 2.73$ & $21.11 \pm 2.89$ & $21.88 \pm 2.41$ & & & \\
\hline & Post-test & $30.11 \pm 3.33$ & $29.44 \pm 2.83$ & $21.88 \pm 2.85$ & 0.001 & 0.025 & 0.001 \\
\hline & Mean difference & 43.38 & 39.45 & 0.2 & & & \\
\hline \multirow{3}{*}{$\begin{array}{c}\text { dynamic leg strength } \\
\text { (number per } 30 \\
\text { seconds) }\end{array}$} & Pre-test & $18.56 \pm 1.66$ & $19.33 \pm 1.80$ & $18.50 \pm 2.81$ & & & \\
\hline & Post-test & $25.33 \pm 2.34$ & $24.44 \pm 2.60$ & $19.50 \pm 2.81$ & 0.001 & 0.016 & 0.001 \\
\hline & Mean difference & 36.47 & 26.43 & 4.05 & & & \\
\hline \multirow{3}{*}{$\begin{array}{c}\text { lower limb muscular } \\
\text { endurance (Number } \\
\text { of steps }\end{array}$} & Pre-test & $30.22 \pm 4.46$ & $30.22 \pm 3.56$ & $28.75 \pm 3.95$ & & & \\
\hline & Post-test & $38.44 \pm 4.92$ & $38.11 \pm 3.91$ & $29.00 \pm 4.00$ & 0.001 & 0.020 & 0.001 \\
\hline & Mean difference & 27.20 & 26.10 & 0.86 & & & \\
\hline \multirow{3}{*}{$\begin{array}{l}\text { cardiorespiratory } \\
\text { function }(\mathrm{cm})\end{array}$} & Pre-test & $353.38 \pm 8.36$ & $351.46 \pm 9.58$ & $352.88 \pm 17.50$ & & & \\
\hline & Post-test & $389.68 \pm 10.50$ & $398.77 \pm 11.80$ & $356.13 \pm 17.44$ & 0.001 & 0.018 & 0.001 \\
\hline & Mean difference & 10.27 & 10.90 & 0.92 & & & \\
\hline \multirow{3}{*}{$\begin{array}{c}\text { dynamic balance } \\
\text { (sec) }\end{array}$} & Pre-test & $8.25 \pm 0.38$ & $7.80 \pm 0.65$ & $7.70 \pm 0.82$ & & & \\
\hline & Post-test & $5.56 \pm 0.40$ & $5.92 \pm 0.56$ & $7.67 \pm 0.80$ & 0.001 & 0.019 & 0.001 \\
\hline & Mean difference & -32.60 & -24.10 & -0.38 & & & \\
\hline
\end{tabular}

*Significant between-group difference; "Significant within-group difference. 


\section{Funding}

The paper was extracted from the $\mathrm{PhD}$. dissertation and extracted from a research project of the first author at the Department of Sports Physiology, Faculty of Sports Science, Hakim Sabzevari University, Sabzevar.

\section{Authors' contributions}

Conceptualization, writing - review \& editing: All authors; Methodology: Roya Askari, Mohsen Damavandi; Investigation: Mehdi Pouyafar, Roya Askari, Ali Maleki; Supervision: Mehdi Pouyafar, Roya Askari; Supervision, funding acquisition: Mehdi Pouyafar.

\section{Conflicts of interest}

The authors declare no conflict of interest. 
This Page Intentionally Left Blank 


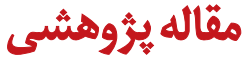

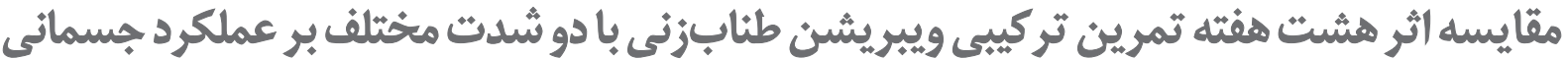

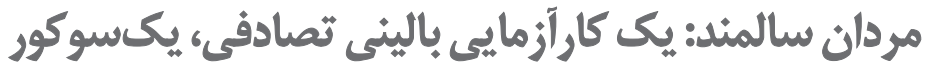

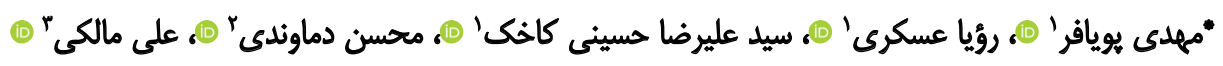

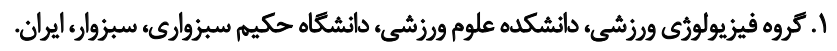

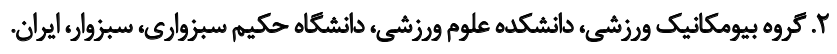

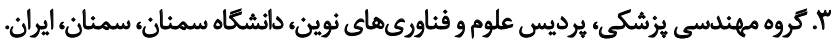

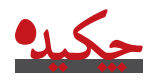

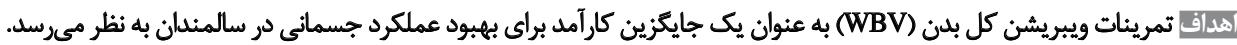

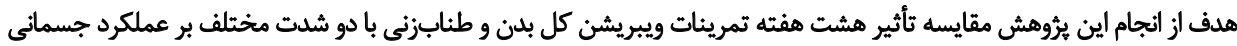

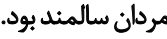

موادوروفي ها

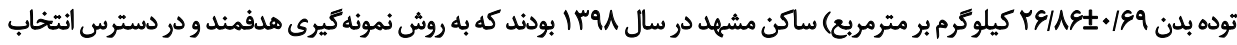

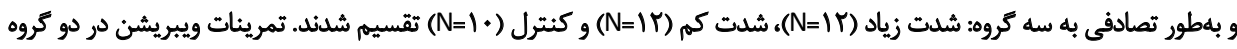

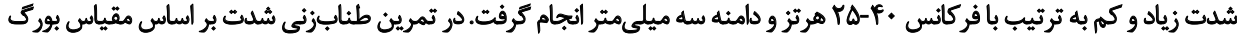

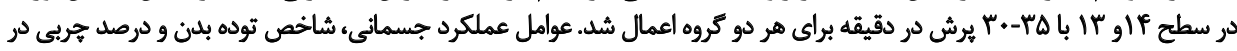

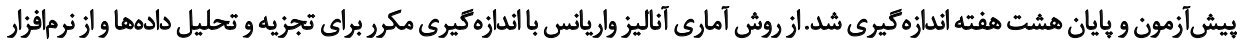
SPSS

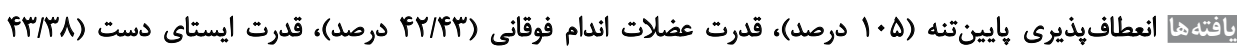

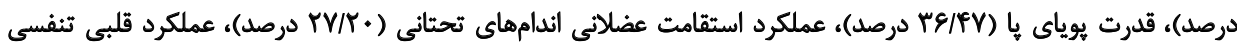

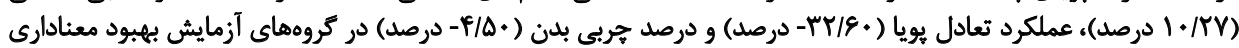

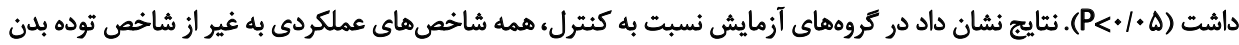

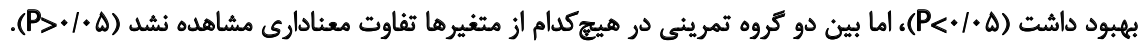

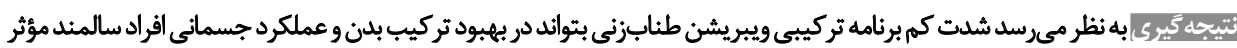

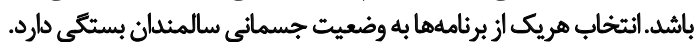

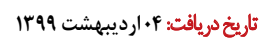

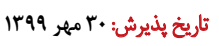

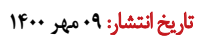

جسمانى بيشتر ميشود و به علت اختلالاتى كه در سيستمهاى

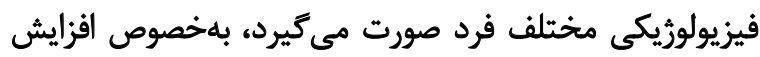

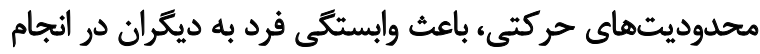

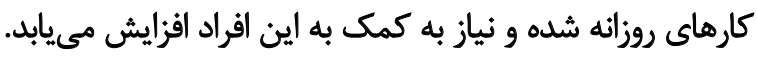

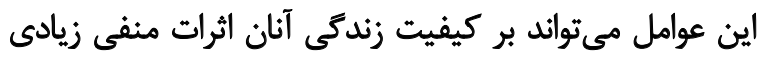

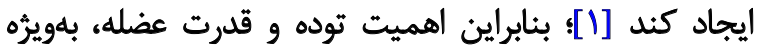

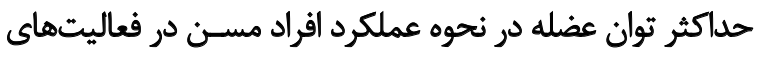

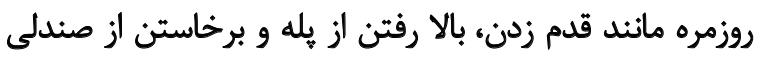

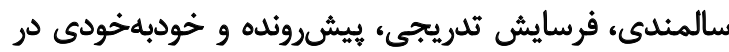

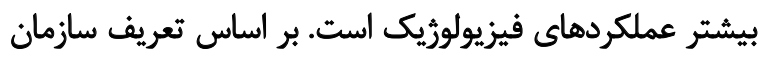

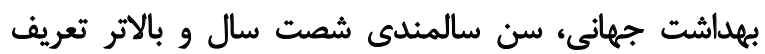

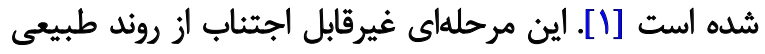

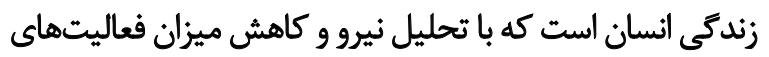

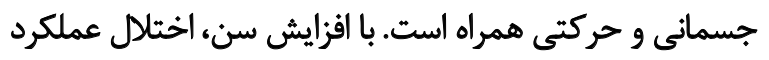

* نويسئده مسئول:

دكتر مهدى يويافر

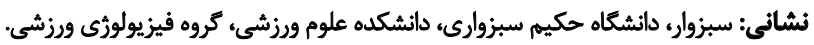

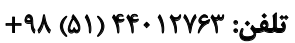
r.askari@hsu.ac.ir بيست الكترونيكي 
جابهجايى) اين نوسانها تعيينكنئده شدت (شـتاب) باركذارى

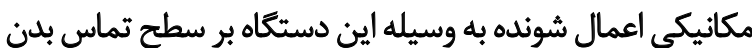

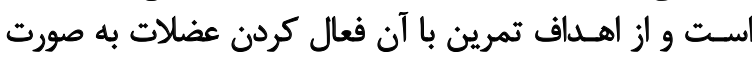

$$
\text { مكانيكى است [1/]. }
$$

برنامه تمرينات ويبريشن در افراد مسن نسبت به افراد جوان و

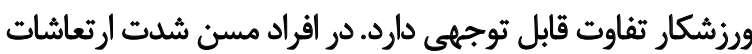

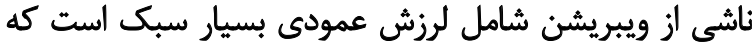

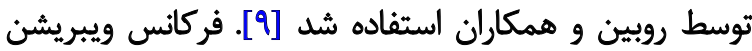

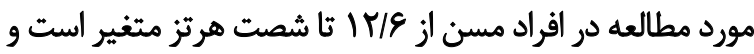

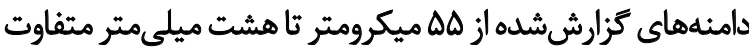

اكرجه فركانس بايينتر از اين محدوده، به ويروه با دامنه

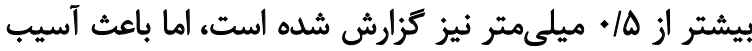

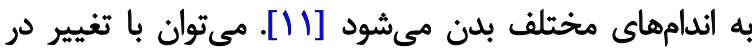

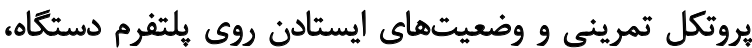

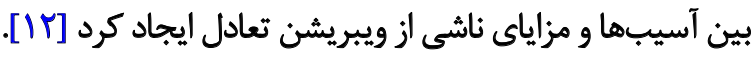

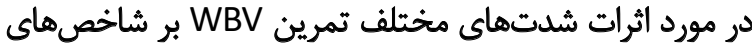

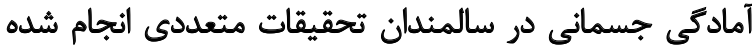
است و يافتههاى كمابيش متناقضى وجود دارد. به طور مثال، بروير و همكاران تمرينات ويبريشن را با فركانس

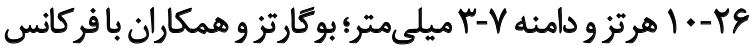

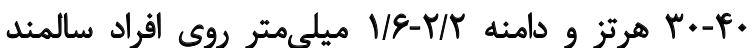

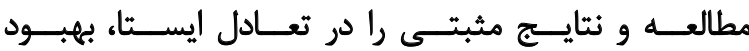

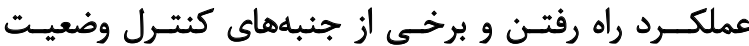

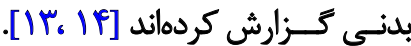

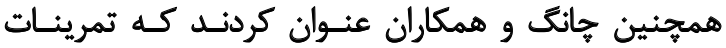

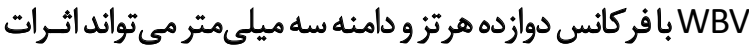

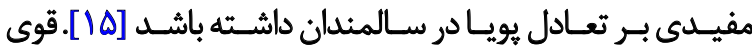

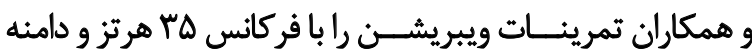

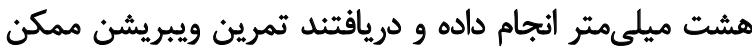

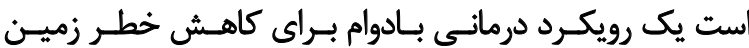

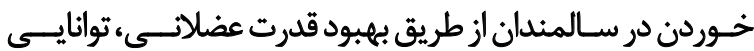

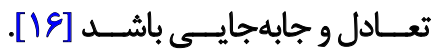

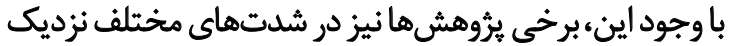

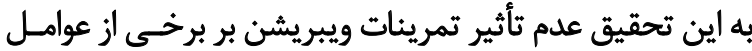

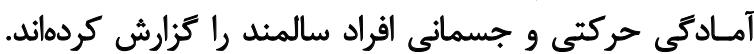

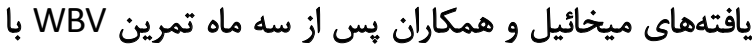

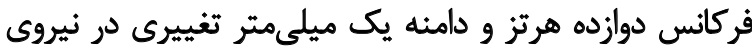

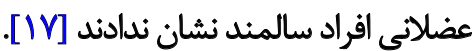

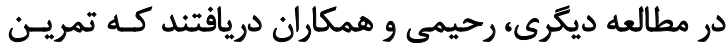

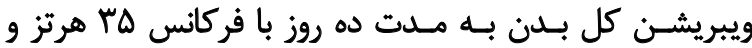

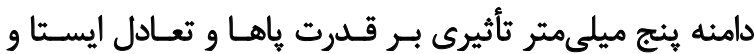

تأثير بسزايى دارد [ب]. بديهى است ضعف عضله و كاهش توانايى

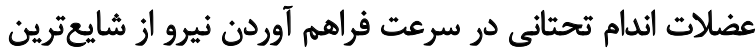

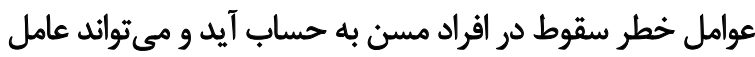

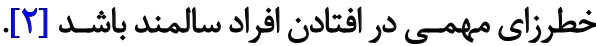
به زمين افتادن يا سقوط يكى از شايعترين و جدى جترين

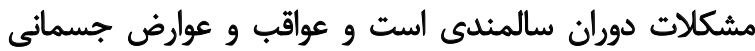

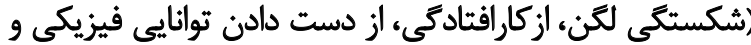

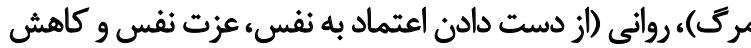
اميد به زندكى) و مادى زيادى دارد دادئ.

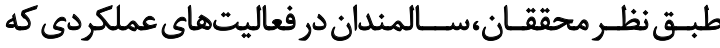

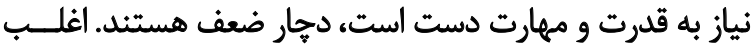

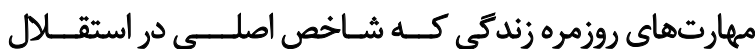

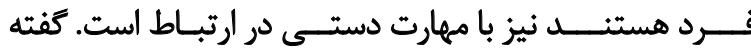

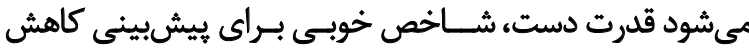

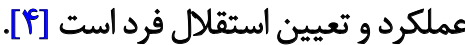

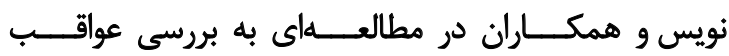

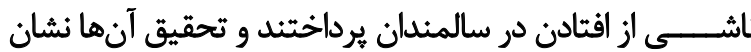

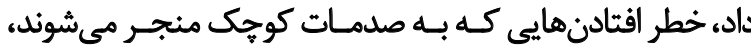

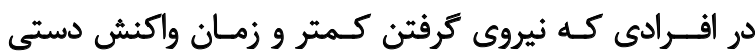

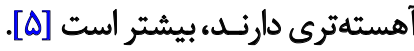

انجام تمرينات سنتى كه روى زمين انجام مي شيوند، براى

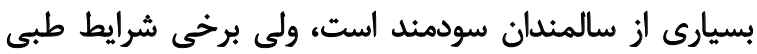

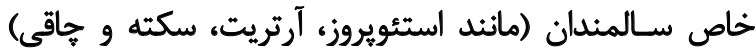

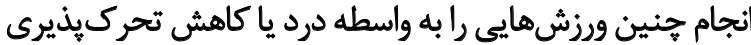

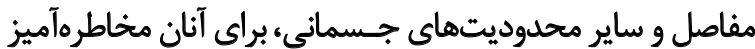
و با رغبت عمومى كمترى روبهرو كرده است يا مانع از انجان انجام اين مانين

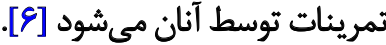

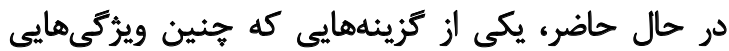

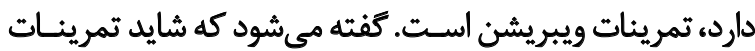

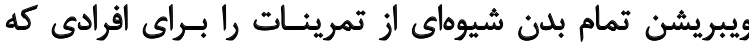

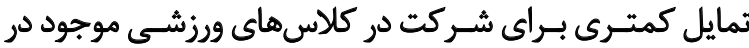

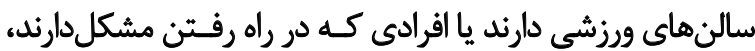

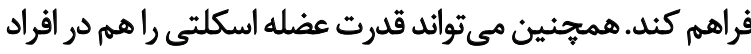

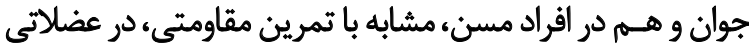

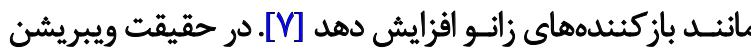

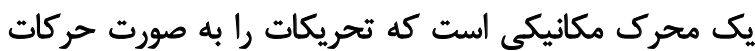
نوسانى و به طور مداوم به كل بدن انتقال مي دهدي

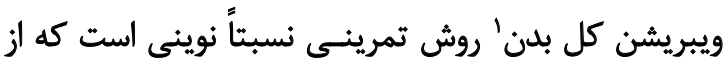

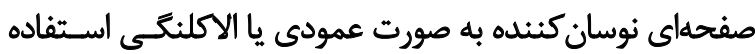
مى كند؛ كـــ تركيب اندازه (مقدار جابهجايى) و سرعت (فركانس

1. Whole-body Vibration (WBV) 


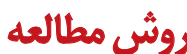

اين تحقيق از نوع نيمهتجربى باطرح ييش و يسآزمون باكروه

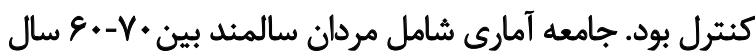

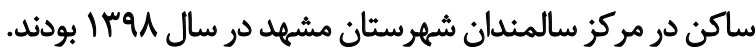
حجم نمونه با استفاده از نرمافزار

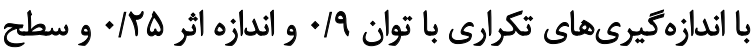

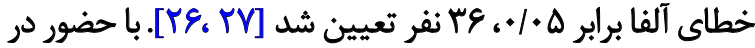

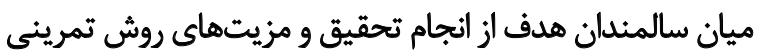

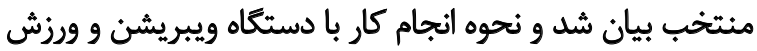

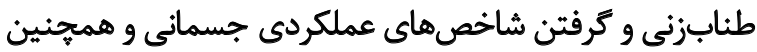
انجام معاينات بزشكى توضيح شاخص داده شد.

شرايط ورود: دارا بودن سطح سلامت عمومى با استفاده از تئي

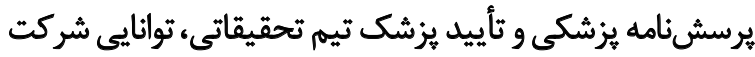

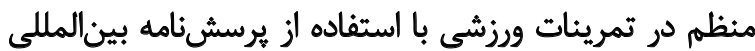

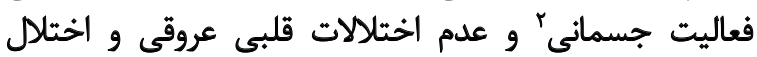

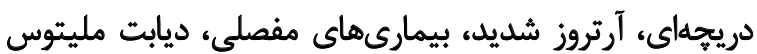

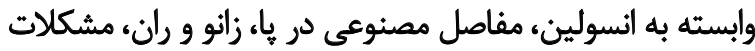

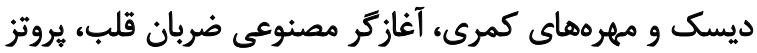

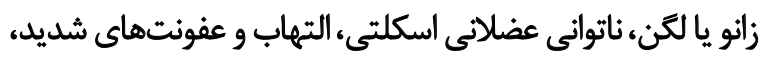

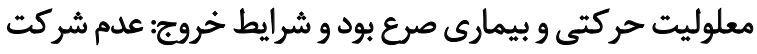
منظم در جلسات تمرينى، مشكلات احتمالى جسمانى و ود دلايل

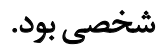

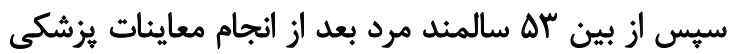

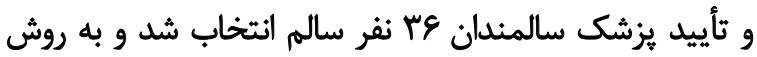

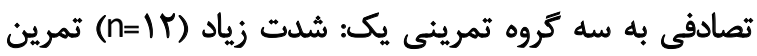

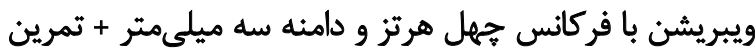

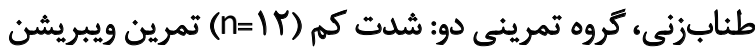

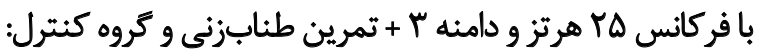

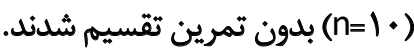

براى تعيين واحدهاي يروهش در سه كروه دوازده نفرى از ئري

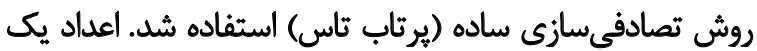

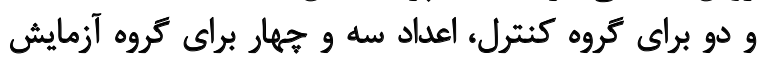

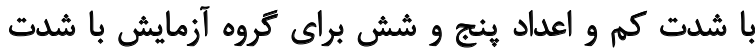

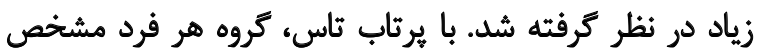

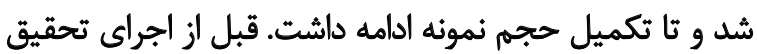

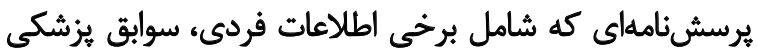

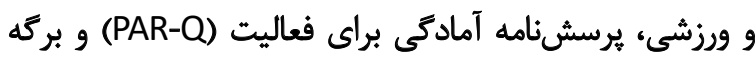

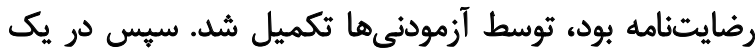

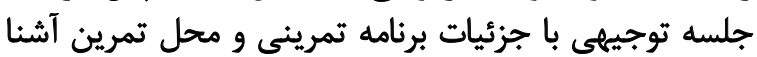

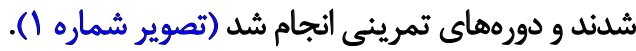

2. The International Physical Activity Questionnaire (IPAQ)

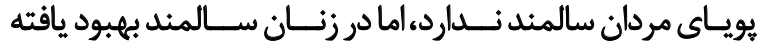

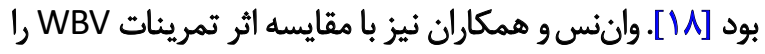

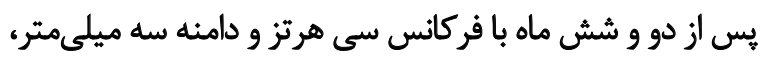

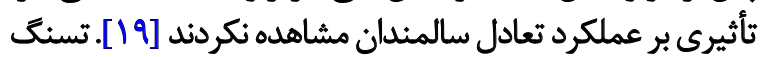

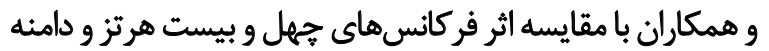

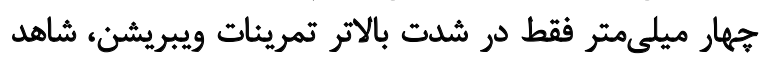

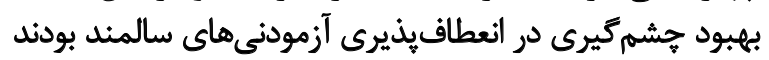

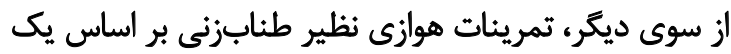

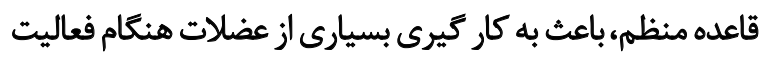

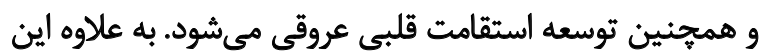

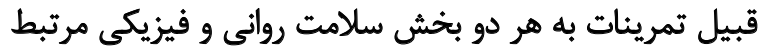

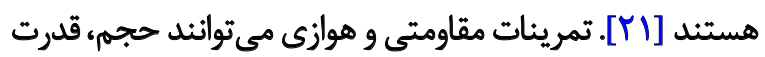

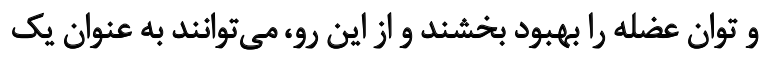

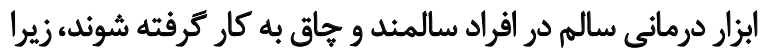

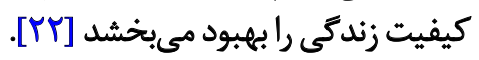

كفته ميشود كه تمرين طنابزنى يكى فعاليت ارزشمند است

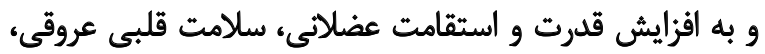

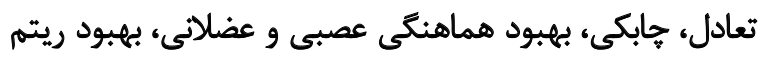

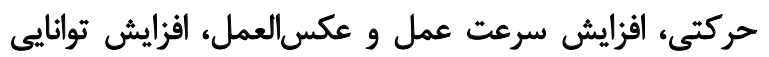

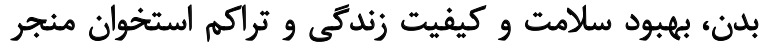

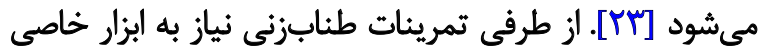

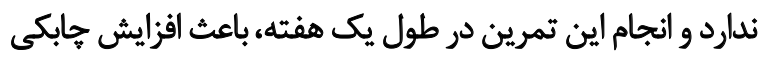

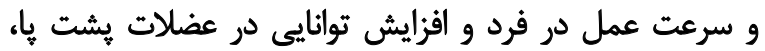

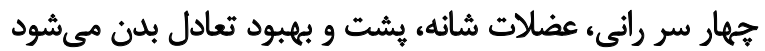

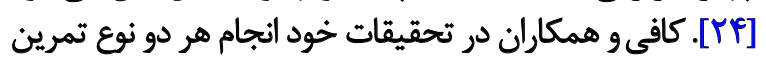

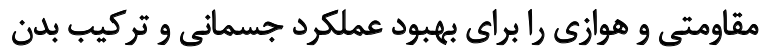
در سالمندان توصيه كردهاند [Tهائ. با توجه به كمخطر و كمهزينه بودن و نياز كمتر به تجهيزات

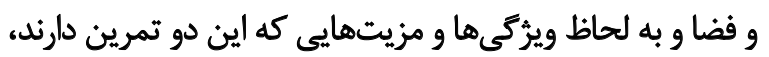

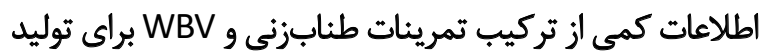

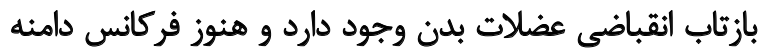

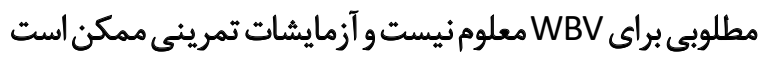

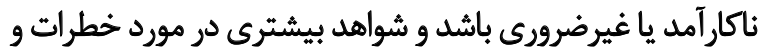

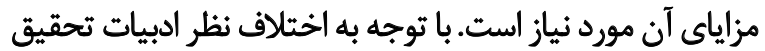

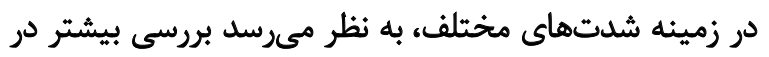

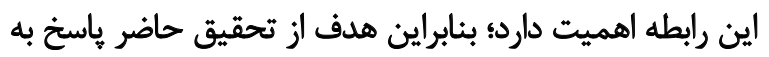

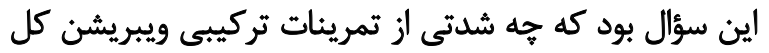

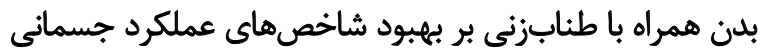

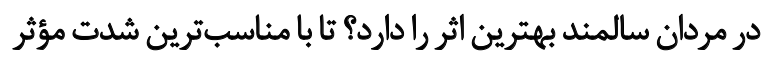

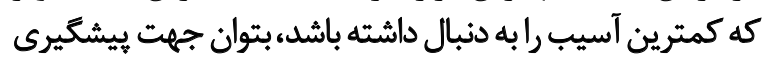

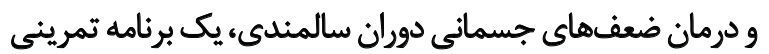
مطلوب و ايمن راطراحى كرد. 
ثبت نام

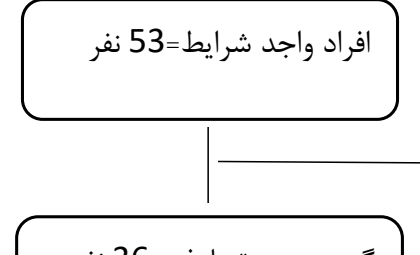

كروه بندى تصادفى=36 نفر

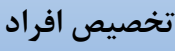

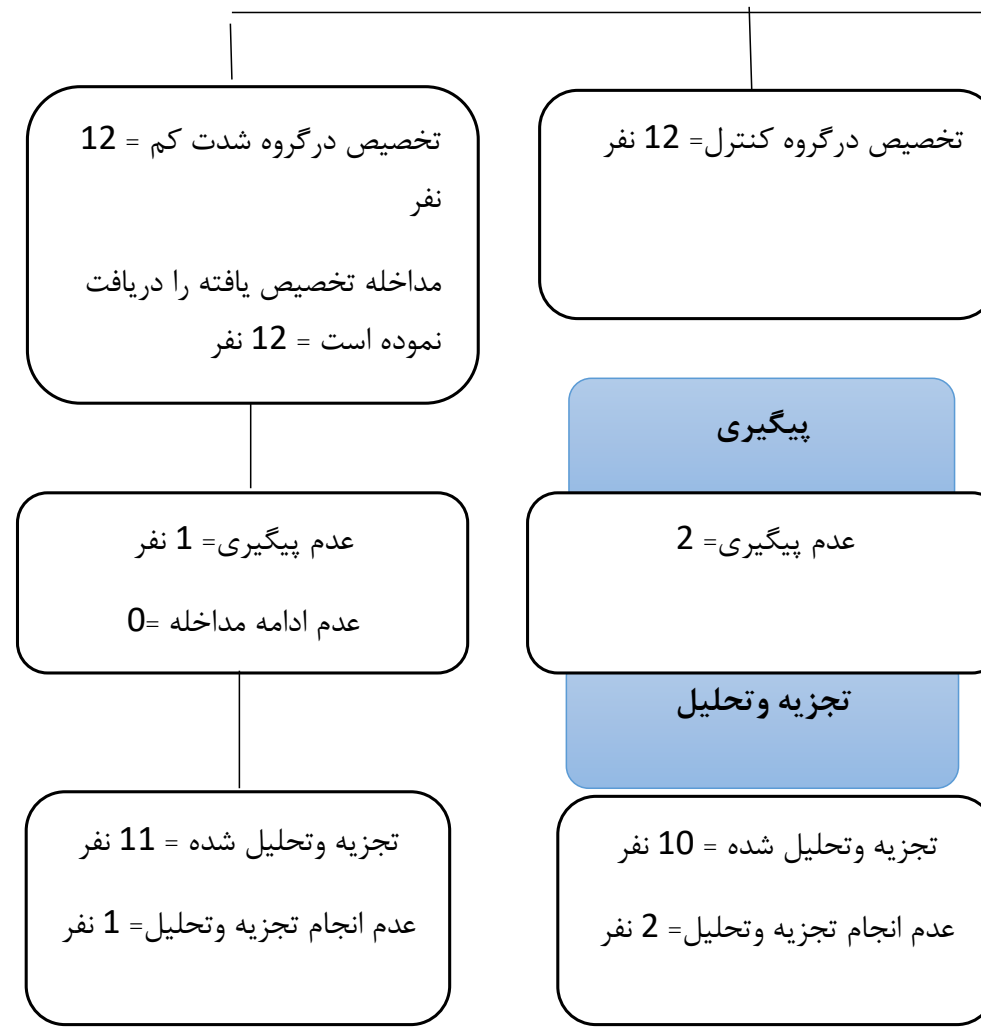

䂪

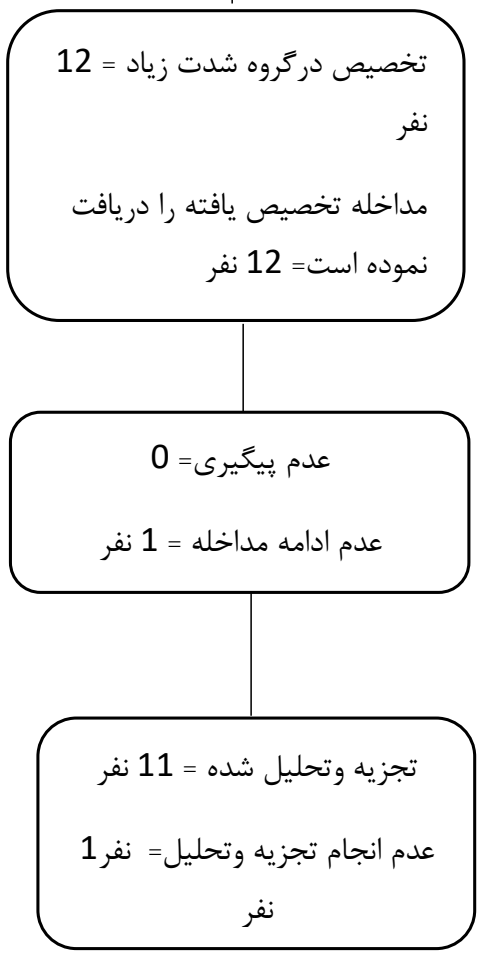

تصوير ا. فلودياكرام كانسورت

تركيب بدن (دستكاه Full Body Composition مدل BF511)

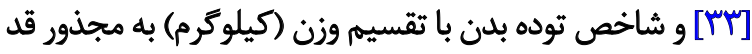

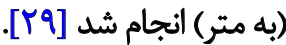

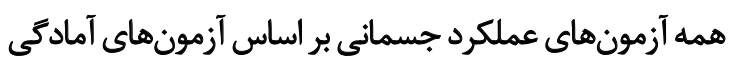

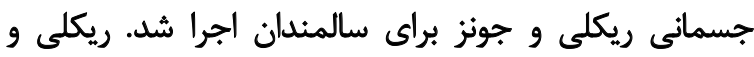

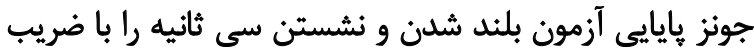

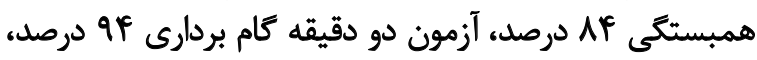

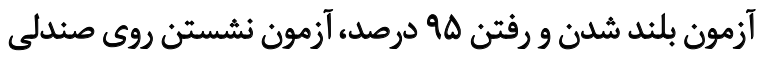

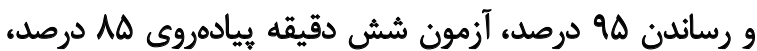

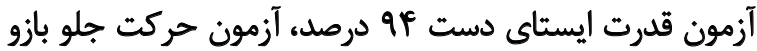

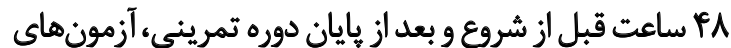

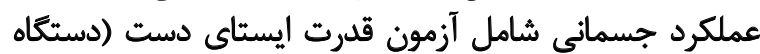

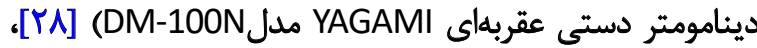

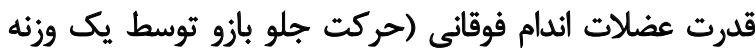

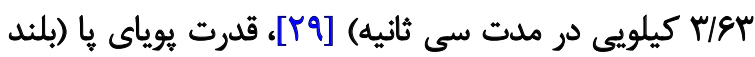

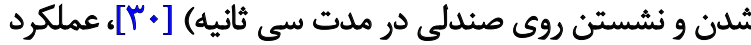

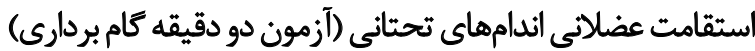

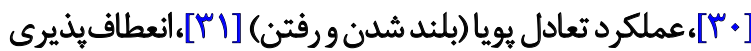

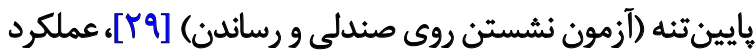

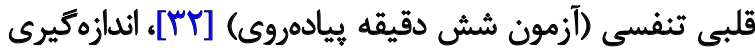


جدول ا. برنامه تمرينات ويبريشن (كروه يك و دو)

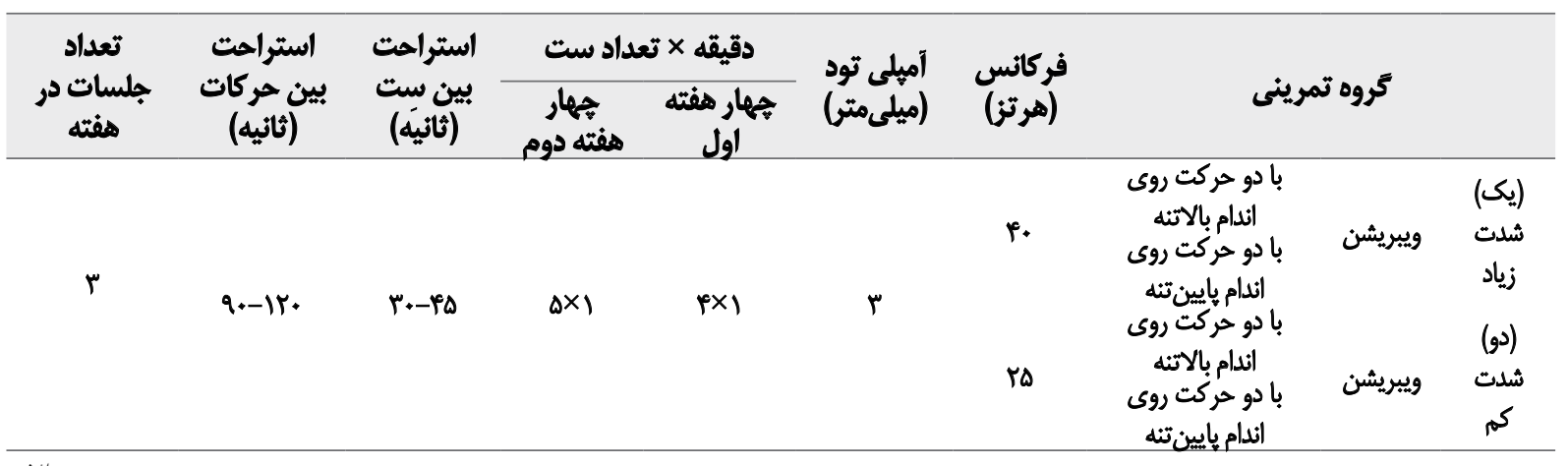

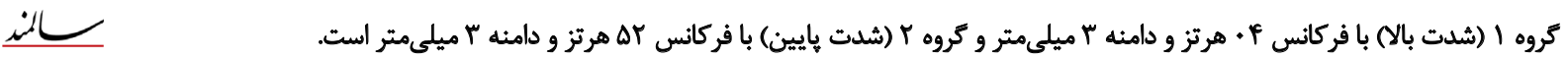

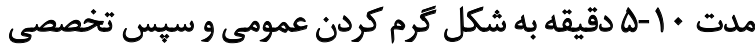

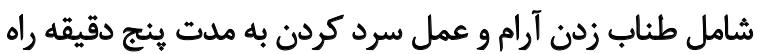

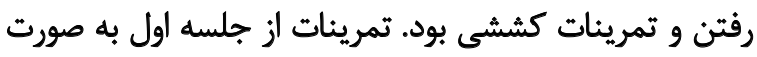

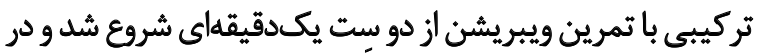

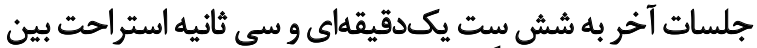

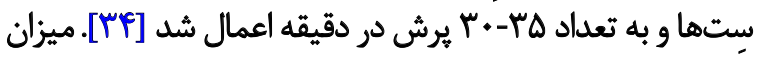

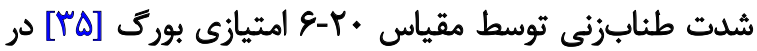

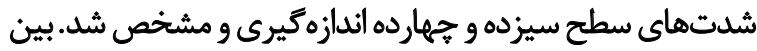
تمرين ويبريشن و طنابزني ينج دقيقه استراحت فعال شامل

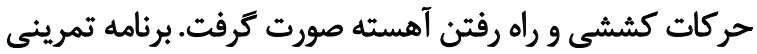

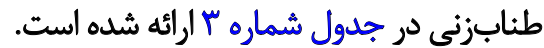

جهت تحليل دادهها از روش آمارى ثحليل واريانس با بادئ

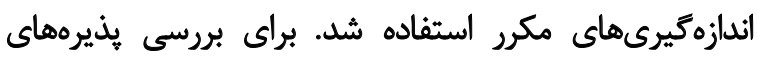

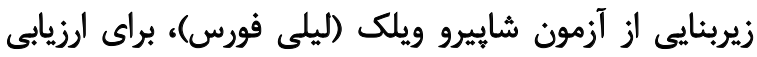

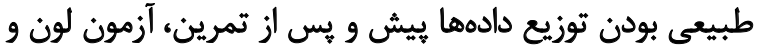

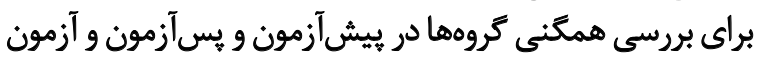
باكس (براى بررسى ثابت بودن ماتريس كورواريانس خطا) اجرا

در متغير هاي انعطاف يذيرى يايينتئه، قدرت يوياي ياء، عملكرد

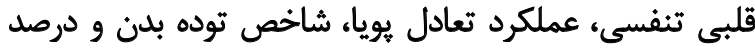

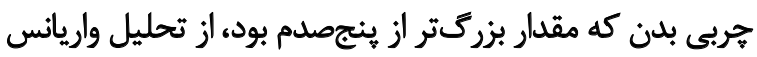

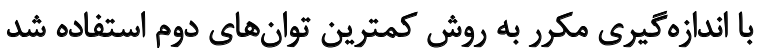

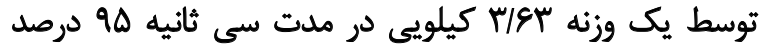

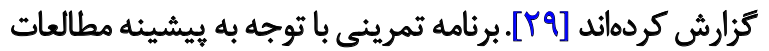

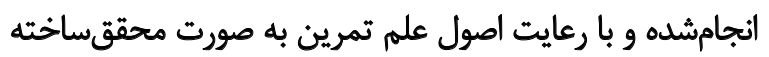

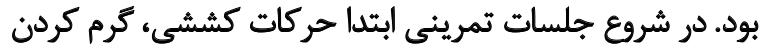
عمومى و كرم كردن اختصاصى اجرا و سيس تمريس تمرين ويبريشن

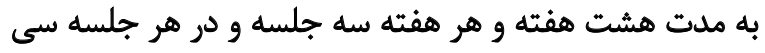

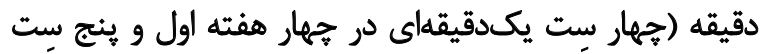

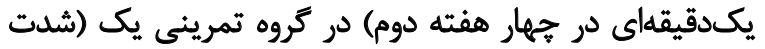
زياد) و كروه دو (شدت كم) اجرا شد. دامنه براى هر دو كروه يكسان، برابر با سه ميلي متر و فركانس

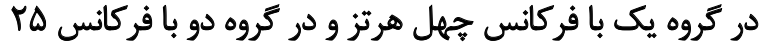

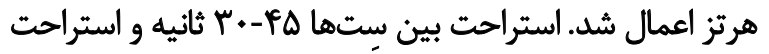

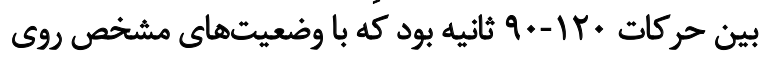

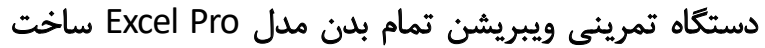

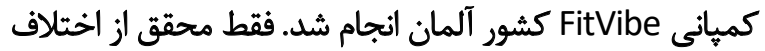

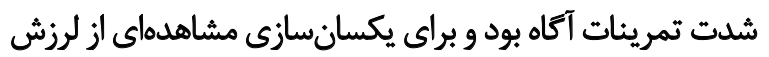

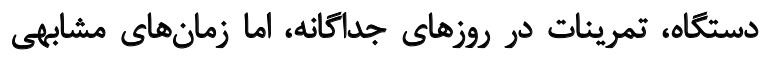

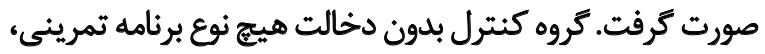
فعاليتهاى خود را مطابق روال قبل انجام مي دادند. برنامه تمرين

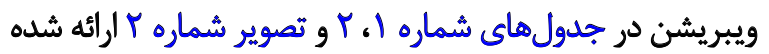
أست. برنامه تمرينى طنابزنى براى سالمندان: عمل گرم كردن به

جدول ז. حركات بالاتنه و يايينتيه

\section{وضعيتهاي مختّلف قرار مراقتن روى دستكاه ويبريشن}

\section{بإيبنتنه}

$$
\text { بم- اسكات ها (حركات خمم و صاف شلن) روى صفحه وييريشن }
$$

\section{بالاتنه}

ا- بلن به حالت شناى كامل و دستها روى صفحه ويبريشن و به حالت ثابت، رومئ.

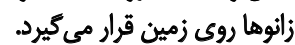

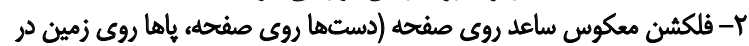
وضعيت بشت به صفحه ويبريشن) (دسن) 


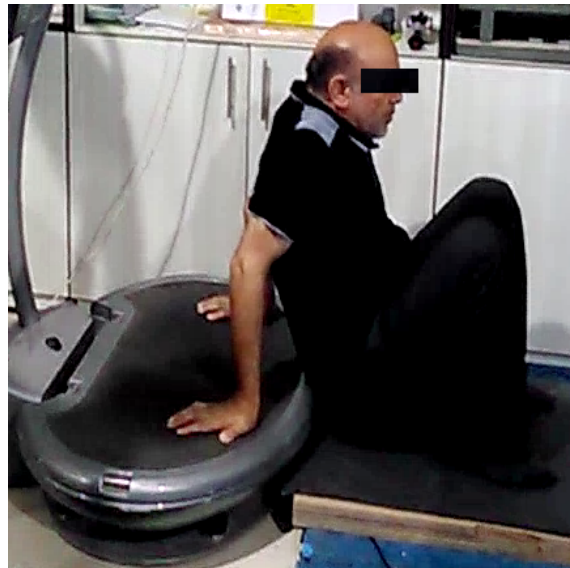

Y- وضعيث

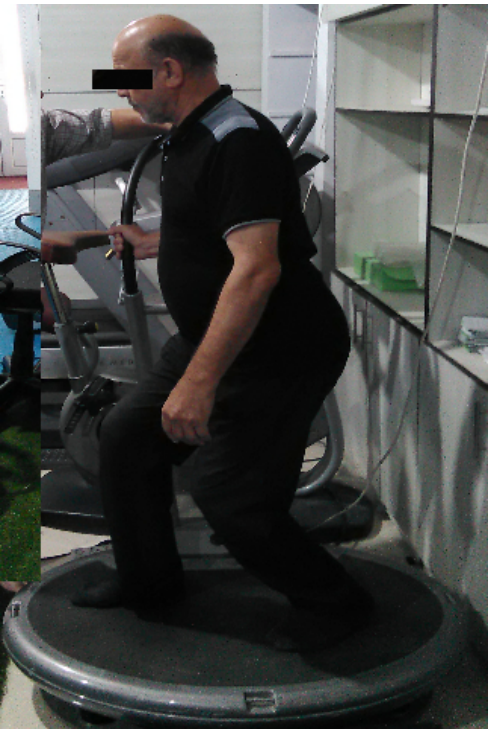

$f=$ وضعيث

الم

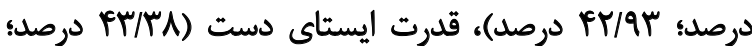

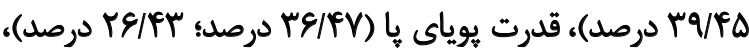

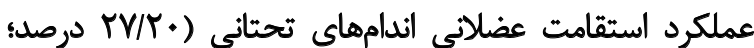

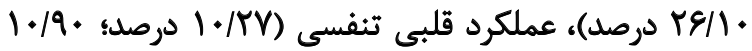

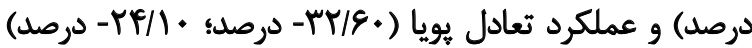

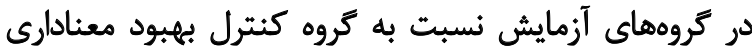

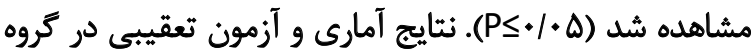

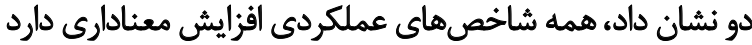

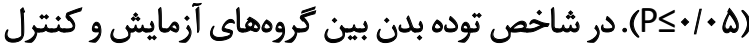

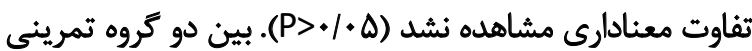

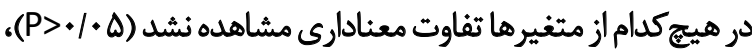

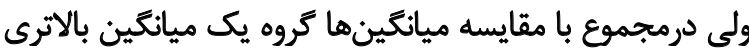

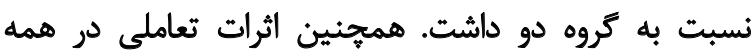

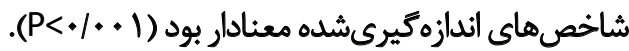

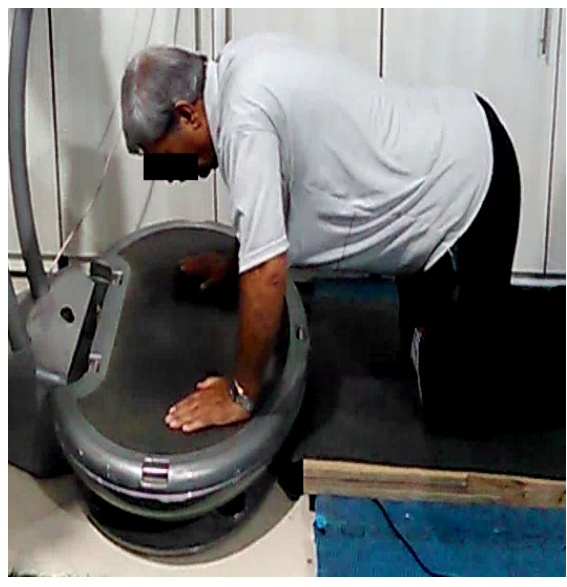

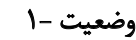

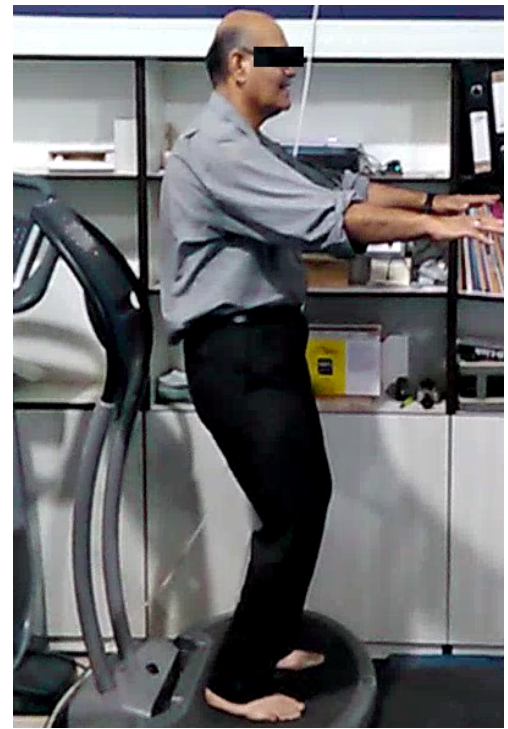

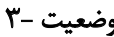

تصوير r. وضعيتهاي مختلف قرار ترفتن روى دسئكاه ويبريشن

و در متغير هاي قدرت عضلات اندام فوقاني، قدرت ايستاي دست

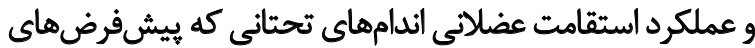

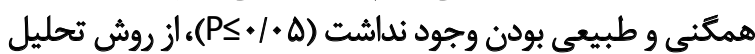
واريانس با اندازههاى مكر وزني دار شده (وزنى) استفاده شده است. در صورت معنادارى براى تعيين محل اختلاف از آزمون

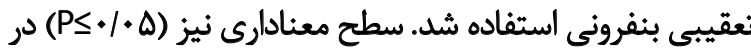

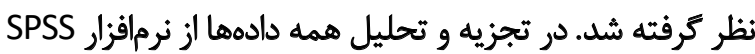

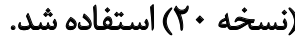

بافتهن

مشخصات آزمودنى ها در جدول شماره F ارائه شده است. نتايج

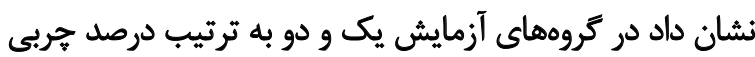

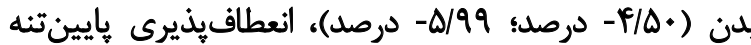

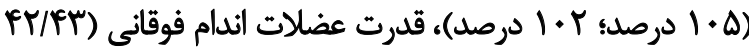


جدول ஈ. برنامه تمرينى طنابزنى

\begin{tabular}{|c|c|c|c|c|c|c|c|c|}
\hline هشتم & هفتم & شُشم & هنجبم & جهارم & سوم & دوم & اول & هفته \\
\hline 8 & 8 & $\Delta$ & $\Delta$ & $p$ & $p$ & $r$ & $r$ & تعلداد سِت \\
\hline 1 & 1 & 1 & 1 & 1 & 1 & 1 & 1 & زمان سِتها (دقيقه) \\
\hline$r$ & r. & $r$ & $r$ & r. & $r$ & $r$. & r. & استراحت بين بتها (ثائيه) \\
\hline$r \cdot-r \Delta$ & $r \cdot-r \Delta$ & $r \cdot-r \Delta$ & $r \cdot-r \Delta$ & $r \cdot-r \Delta$ & $r \cdot-r \Delta$ & $r \cdot-r \Delta$ & $r \cdot-r \Delta$ & تعلداد برش در دقيقه \\
\hline r & r & r & r & $\mu$ & r & $r$ & r & تعلداد جلسات در هفته \\
\hline
\end{tabular}

مى تواند روشى مناسب جهت دستيابى به اين اهداف در افراد

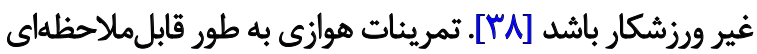

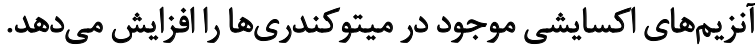

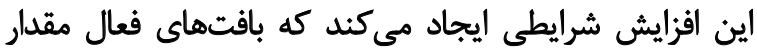

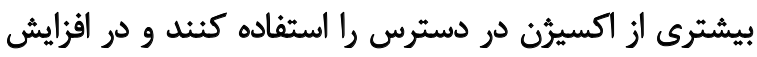

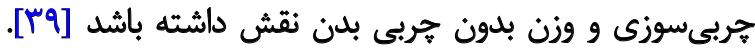

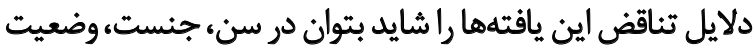

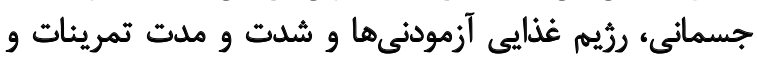
همجنين روش اندازهيرى متغيرها أذكر كرد.

طبق جدول شماره ه نتايج مطالعه حاضر نشان داد، كروههاى

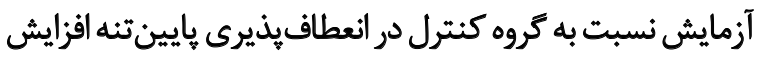

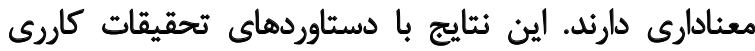

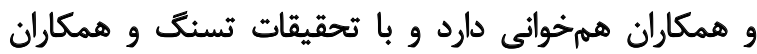

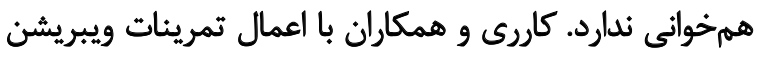

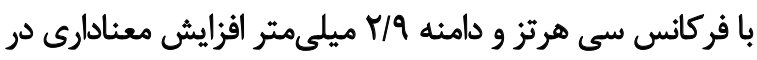

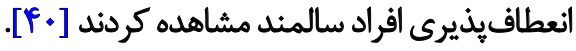

تسنك و همكاران با انجام تمرينات ويبريشن با فركانسهاى

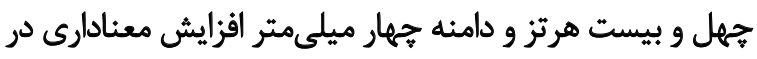

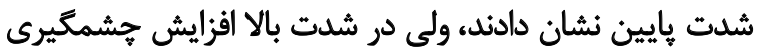

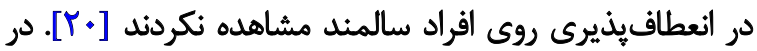

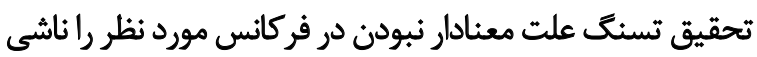

نتايج مطالعه حاضر نشان داد، كروههاى آزمايش نسبت به كروه

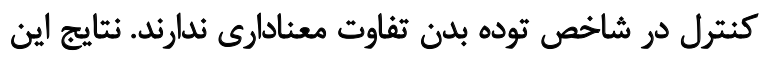

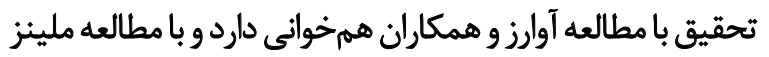

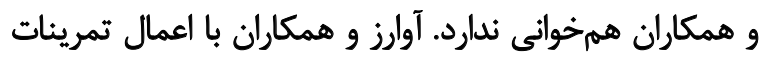

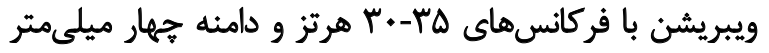

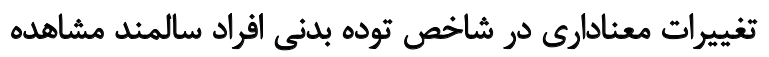

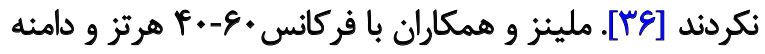

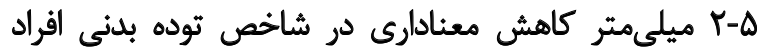

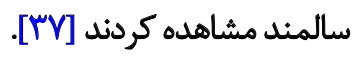

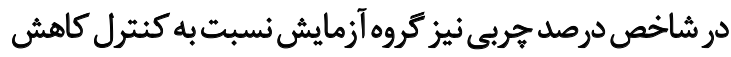

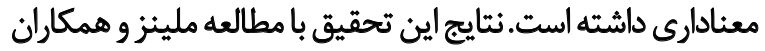

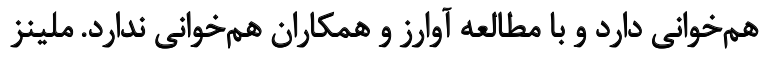

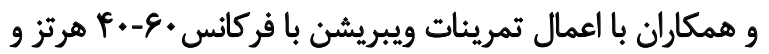

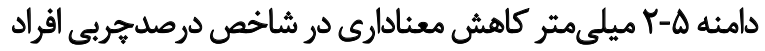

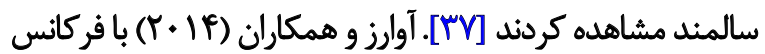

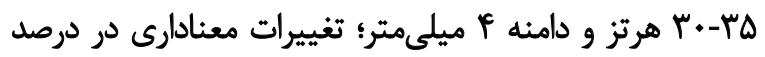

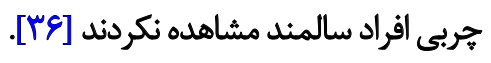

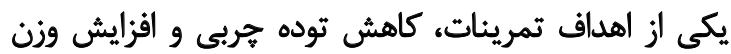

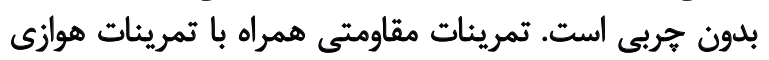

جدول f. مشخصات آزمودنىها در دو كروه قبل از انجام دوره تمرينى

\begin{tabular}{|c|c|c|c|}
\hline \multicolumn{3}{|c|}{ ميانكين |انحرافمعيار } & \multirow{2}{*}{ شُخص } \\
\hline كروه كثترل & r Fروه & كروه ا & \\
\hline$\varepsilon \Delta / q Y \pm F / F \gamma$ & 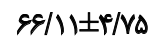 & 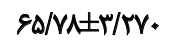 & سن (سال) \\
\hline IENIYIF/9Q & $\mid E V / A q \pm r / \& \Delta$ & $|\gamma| V A \pm r / \cdot V r$ & قد (سائتيمتر) \\
\hline$V \Delta / A f \pm E / M F T$ & $V \varepsilon / F \in \pm \mu / F \Delta$ & VNAr \pm T/AqA & وزن (كيلوكرم) \\
\hline$r E / N A \pm V / \cdots A$ & TWMI./ATE & relFat. $10 F r$ & شاخص توده بلن (كيلوكرم بر مترمربع) \\
\hline
\end{tabular}


جدول ه. دادهاي توصيفى شاخص هاى عملكرد جسمانى

\begin{tabular}{|c|c|c|c|c|c|c|c|}
\hline \multirow{2}{*}{$\begin{array}{l}\text { اثرزمان } \\
\text { ×ووه }\end{array}$} & \multirow{2}{*}{ اثرئروهل } & \multirow{2}{*}{ زاثر أرامل } & \multicolumn{3}{|c|}{ ميانكين土انحراف معيار } & \multirow{2}{*}{\multicolumn{2}{|c|}{ bogs }} \\
\hline & & & تَّوه كتترل & تروه تمرينى T & تروه تمرينى & & \\
\hline \multirow{3}{*}{$.1 . .1$} & \multirow{3}{*}{ - /PA. } & \multirow{3}{*}{$.1 . .1$} & rE/VA $\pm 1 / .$. & $r V / 1 \pm \cdot / \Delta r$ & $r \& / g q \pm \cdot / \Delta \&$ & ييش أزمون & \multirow{3}{*}{ (كيلوكرم بر مترمربع) } \\
\hline & & & $r g / M+ \pm 1 / \cdot r$ & $r E / A q \pm \cdot / \Delta T$ & RE/FA土+/OY & يسآزمون & \\
\hline & & &.$- / 18$ & $-\cdot|A|$ & $-\cdot / N A$ & درصد ثغييرات & \\
\hline \multirow{3}{*}{$1 \cdot .1$} & \multirow{3}{*}{.1 .19} & \multirow{3}{*}{$.1 . .1$} & KE/MYII/TH & $r \Delta / \Delta r \pm \cdot / q$. & $r \Delta / r+ \pm 1 / / V$ & يبش أزمون & \multirow{3}{*}{ درصد هربيى } \\
\hline & & & 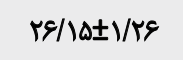 & 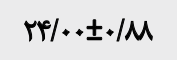 & $r e / 19 \pm$ /IP & يس آزمهون & \\
\hline & & &.$- / M$ & $-ه / 99$ & $-\psi / \Delta$. & درصد تغييرات & \\
\hline \multirow{3}{*}{$.1 .+1$} & \multirow{3}{*}{$.1+11$} & \multirow{3}{*}{$.1 . .1$} & $-8 / 8 Y \pm 1 / 99$ & $-V / \wedge 9 \pm / / A r$ & $-V / \wedge Q \pm) / Q$ & بيش أزمون & \multirow{3}{*}{ 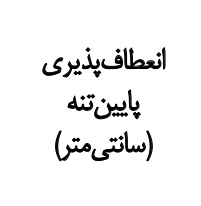 } \\
\hline & & & $-g / N \Delta \pm Y / M r$ & $\cdot / r T \pm 1 / R \Lambda$ & $. / T F \pm T / \Delta$ & يس آزهمن & \\
\hline & & & 1/a & $1 . r$ & $1+0$ & درصد تنييرات & \\
\hline \multirow{3}{*}{$.1 . .1$} & \multirow{3}{*}{.1 .18} & \multirow{3}{*}{$.1 . .1$} & IVIY $\pm / M$ & WAq土Y/.1 & $19 / 1 \pm r / 81$ & ييش آزمون & \multirow{3}{*}{ قلدرت عضلات أندام } \\
\hline & & & 1Q/VDET/TT & $T V / \cdot \pm \pm r / T \cdot$ & $T V / 1 I \pm r / 10$ & يسآزمون & \\
\hline & & & r/Rq & PT/qT & Per/er & درصد تغييرات & \\
\hline \multirow{3}{*}{.1 .01} & \multirow{3}{*}{$.1 . Y A$} & \multirow{3}{*}{$.1 . .1$} & TI/MEY/FI & $r / / 1 \pm r / 19$ & $M / / . \pm r / M r$ & ييش أزمون & \multirow{3}{*}{ قلرت ايسيتاي ديست } \\
\hline & & & TI/AN土T/AD & rq/PFET/Ar & $r \cdot / 11 \pm m / m$ & يس آزّمن & \\
\hline & & & $\cdot \pi$ & rq/Fa & N & درصد تنييرات & \\
\hline \multirow{3}{*}{$.1 . .1$} & \multirow{3}{*}{$+1+18$} & \multirow{3}{*}{$+1++1$} & WA.EY/EV & $19 / \pi m \pm 1 / 1$. & WAF士VIFE & ييش آزّمون & \multirow{3}{*}{ 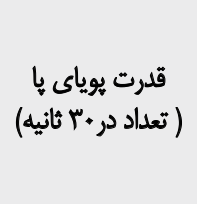 } \\
\hline & & & $|V / \Delta \cdot \pm r / A|$ & $r F / R T \pm r / g$. & $r \Delta / M T \pm Y / M r$ & يسأزّمون & \\
\hline & & & $\varphi / . \Delta$ & re/er & re/pr & درصد تغييرات & \\
\hline \multirow{3}{*}{$.1 . .1$} & \multirow{3}{*}{$.1 . r}$. & & YNVDET/QD & $r \cdot / M T \Psi / \Delta F$ & $r \cdot / T T \pm F / F E$ & ييش آزمون & \\
\hline & & $.1 .+1$ & 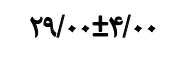 & $r N(1 \pm r / q)$ & rNPFEF/ar & يسآزهون & اندام تحتاني \\
\hline & & & $\cdot / N C$ & re/l. & $r V / r+$ & درصد تغييرات & \\
\hline & & & ror/ALEIV/A. & YQI/FE & 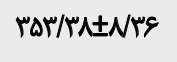 & ييشآزمون & \\
\hline $.1 \cdots 1$ & $+1+11$ & $.1 . .1$ & $r \Delta S / I Y \pm I Y / P F$ & rquW $\pm 11 / 1$. & พАปรภ $\pm 1+/ \Delta$. & نِ & قلبى-تنفسى \\
\hline & & & ./AY & 1.19 . & $1 . / r V$ & درصد تغييرات & 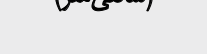 \\
\hline & & & $V / V \cdot \pm \cdot / A Y$ & $V / A \cdot \pm \cdot / 80$ & $N r \Delta \pm / / R A$ & يريشأزمون & i) Sha \\
\hline .1 .01 & .1 .19 & .1 .01 & $V / E V \pm . / A$. & $\Delta / q T \pm=/ \Delta F$ & $\Delta / \Delta F \pm / F$. & يسأزمون & تعادل يويا \\
\hline & & &.$- / \mu \Lambda$ & $-r \varphi / l$. & $-r T / \varepsilon$. & درصد تغييرات & \\
\hline
\end{tabular}


سادهى تحمل وزن، بهبود زيادى در عملكرد و ظرفيت دستها

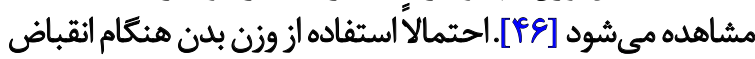

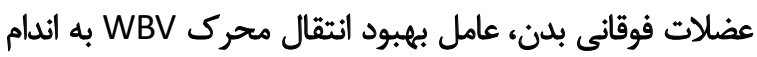

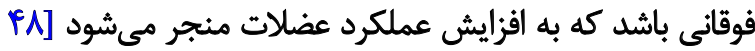

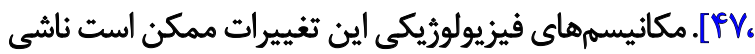

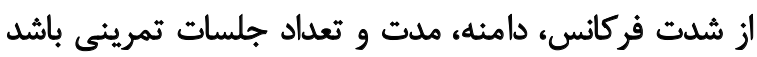

شاخص استقامت عضلاتى اندام تحثاني نيز در كروهمائ

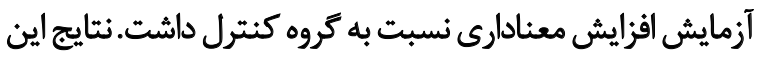

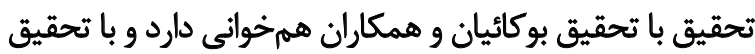

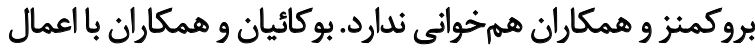

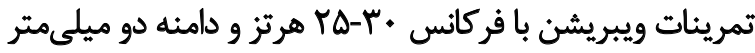

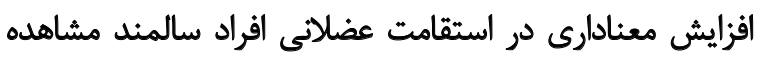

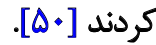

بروكمنز و همكاران با اعمال تمرينات ويبريشن با فركانس

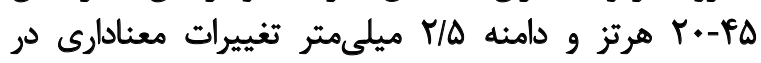

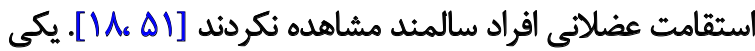

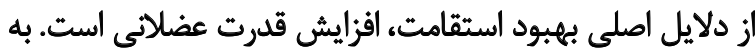

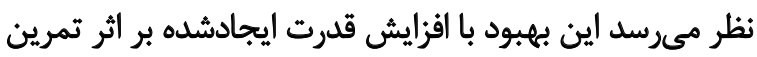

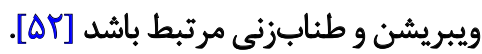

ويبريشن اعمالشده بر تاندونها و عضلات باعث تحريك

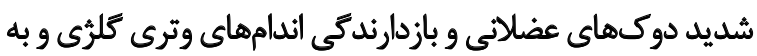

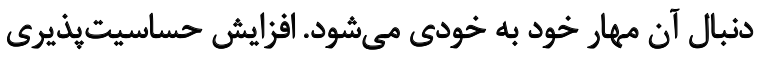

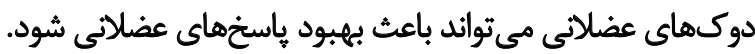

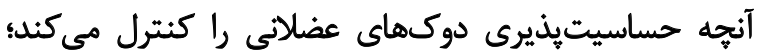

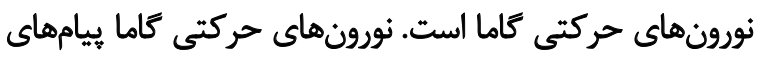

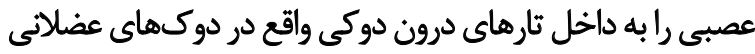

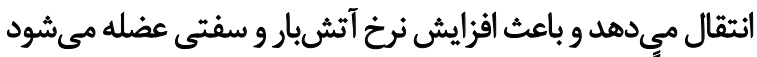

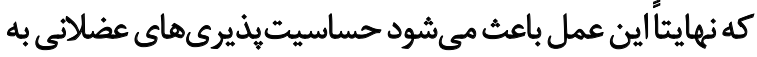

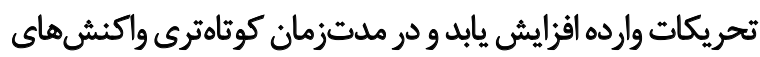

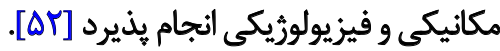

همين طور شاخص عملكرد قلبى تنفسى در كروههاى آزمايش

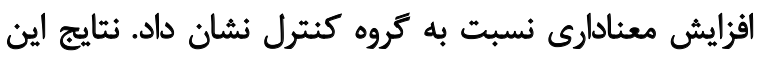

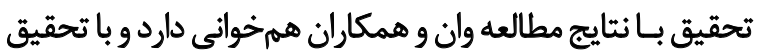

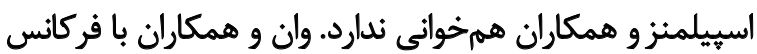

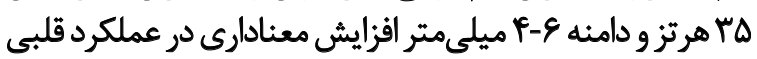
تنفسى افراد سالمند مشاهده كردند [هاميند

اسيّيلمنز و همكاران با اعمال تمرينات ويبريشن با فركانس

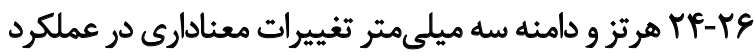

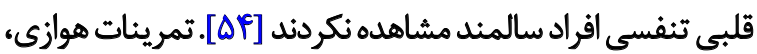

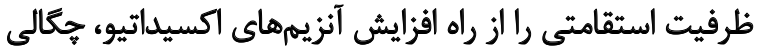

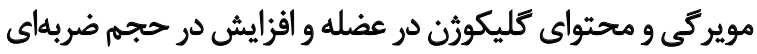

از اختلاف در هاسخ تطبيقى عصبى عضلانى در افراد مسن زن و

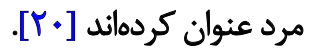

در مطالعه حاضر شدت تمرين در هر دو فركانس در دامنا دامنه

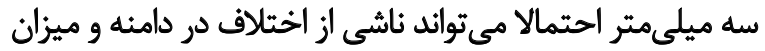

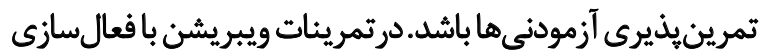

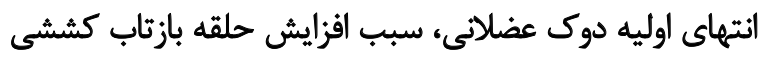

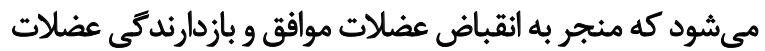

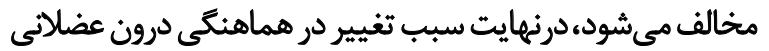

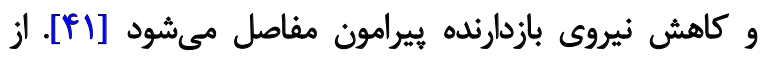

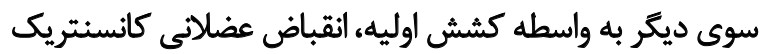

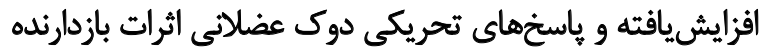

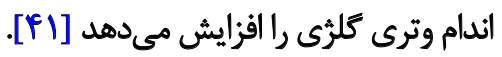

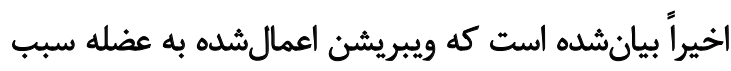

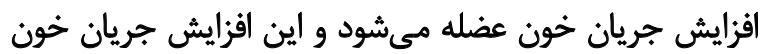

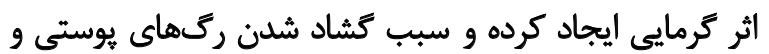

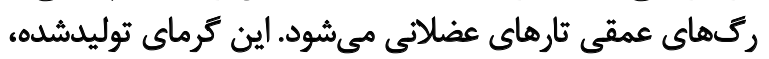

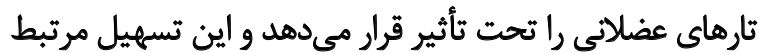

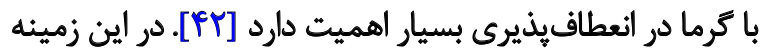

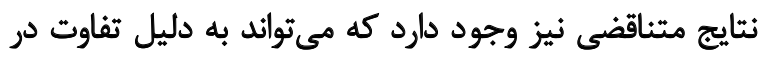

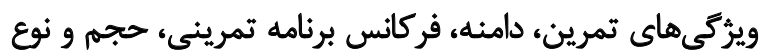
تمرين يا سطح عملكردى افراد سالمند باشد. فرين

نتايج مطالعه حاضر همجنين نشان داد شاخصهائ قدرت

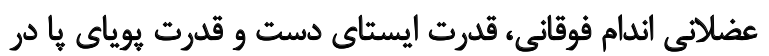

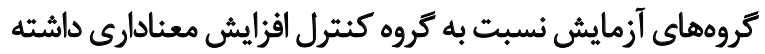

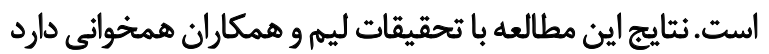

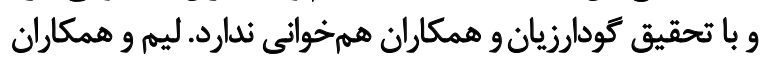

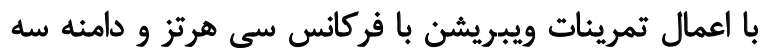

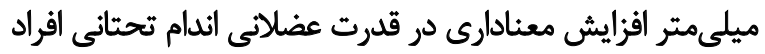

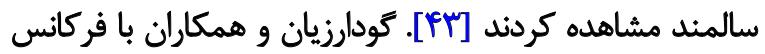

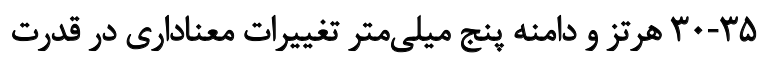

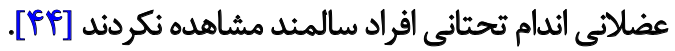
تمرين ويبر يشن در افزرايش توان و قدرت مؤثر است و مكانيسم

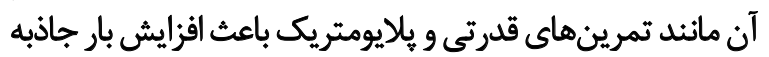

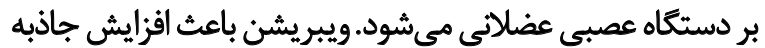

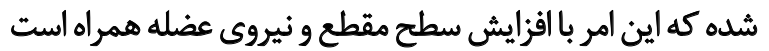

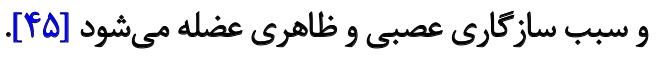
افزايش حساسيتيذيرى دوكهاى عضلاتى و فراخوانى بيشتر

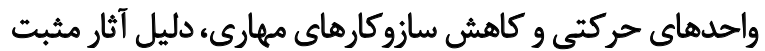

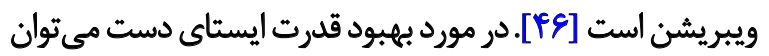

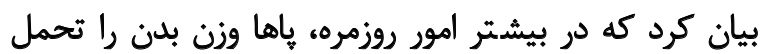

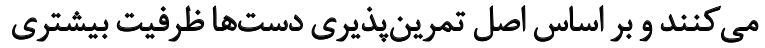

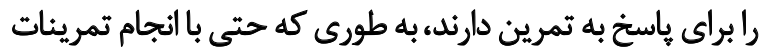




\section{نتيجهيَيرى نهايیى}

نتايج اين مطالعه نشان داد كه تمرينات ويبريشن كل بلد بلن

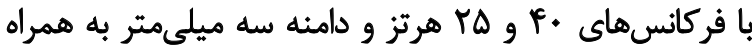

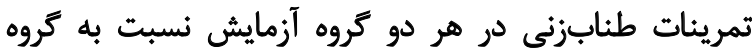

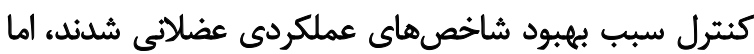

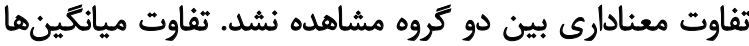

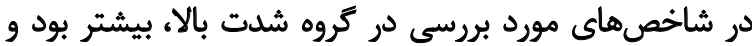

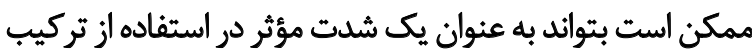
تمرينات ويبريشن طنابزنى توصيه شود.

با توجه به اينكه هر دو شدت تمرينى آثار مثبتى بر شاخصهاي

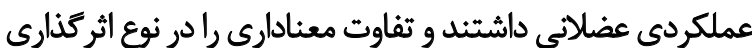

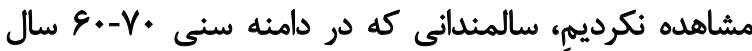

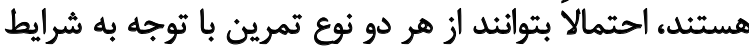

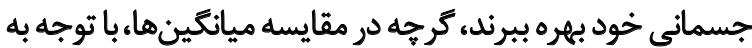

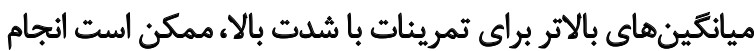

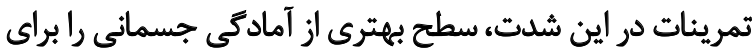

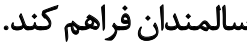

از لحاظ كاربردى مردان سالمندى كه از سلامت جسمانى

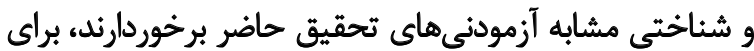

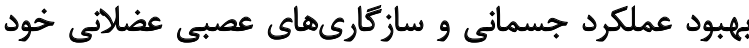

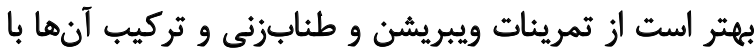

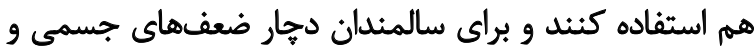

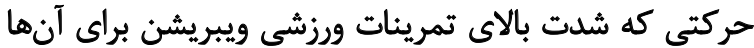

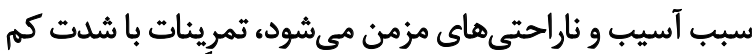

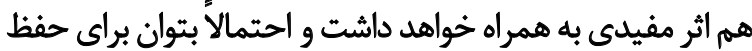

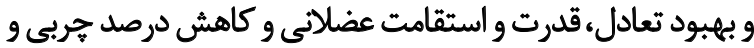

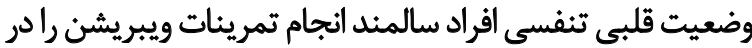

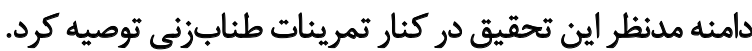
همجنين يكى از مزاياى اين مطالعه استفاده از طنابزنى در

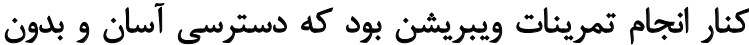

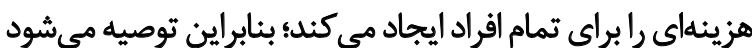

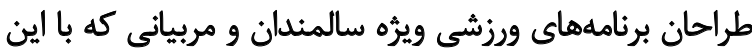

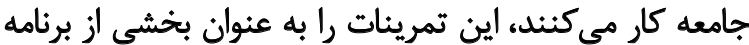
تمرينى آنها قرار دهند.

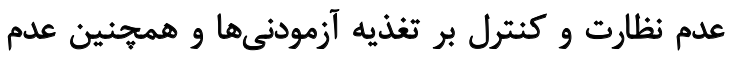

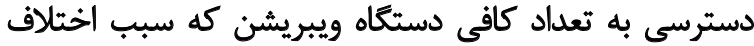

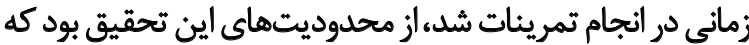
ممكن است نتايج را تحت تأثير قرار داده باشد باشد.

\section{يشئهادات براي يثوهشهاي آيثده}

و كاهش در ضربان قلب بهبود ميدهد و تمرينات مقاومتى نيز

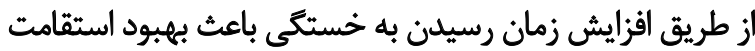

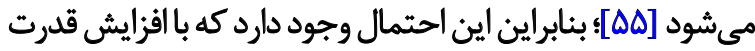

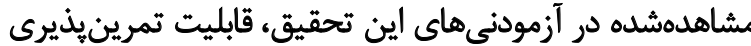

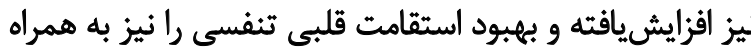

داشته است [IV] ت نئ.

عملكرد تعادل نيز در كروههاى آزمايش نسبت به كروه كنترل

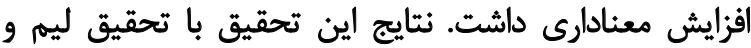

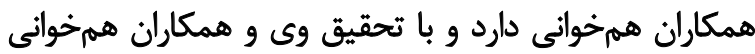

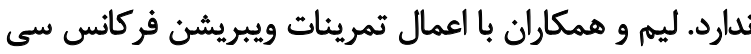

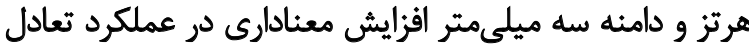

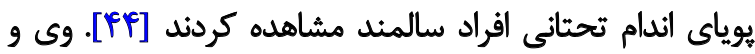

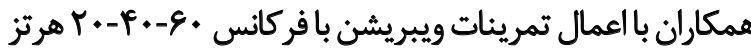

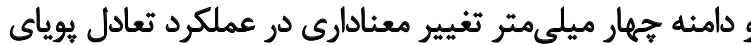

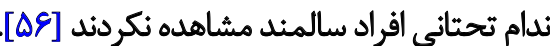

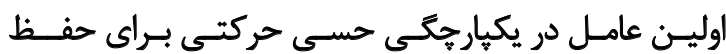

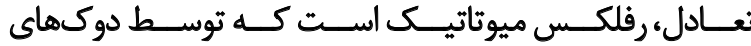

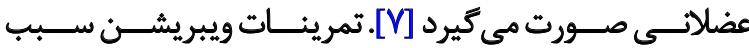

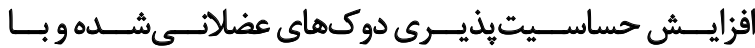

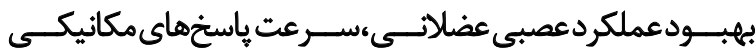

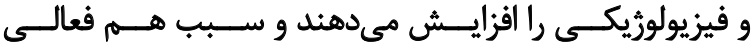

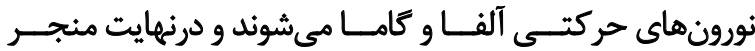

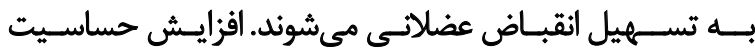

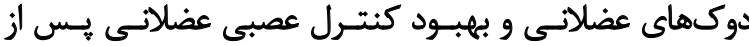

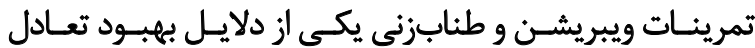

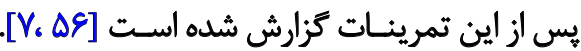

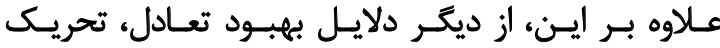

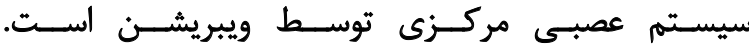

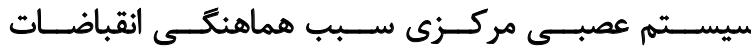

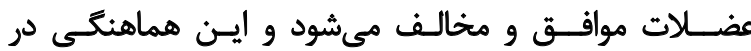

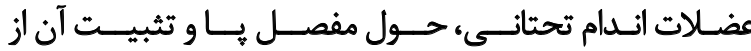

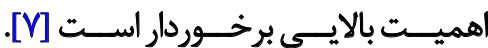

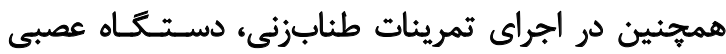

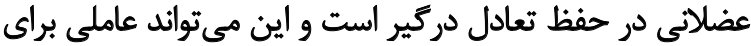

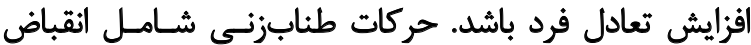

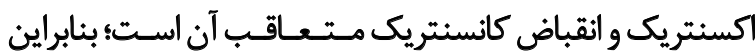

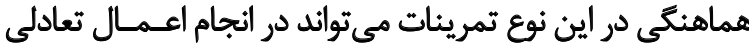

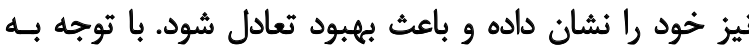

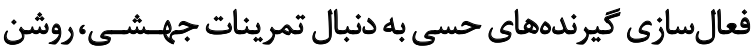

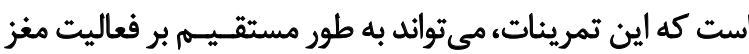

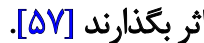

ا. با توجه به اينكه افزايش قدرت و سازكارى اوليه و بهبود 


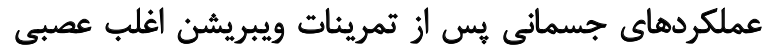

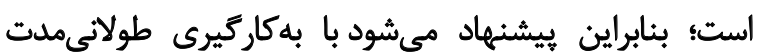

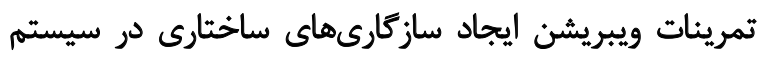
عصبى عضلانى بررسى شود.

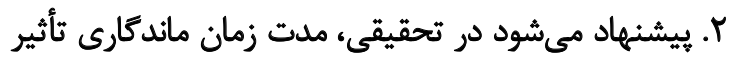

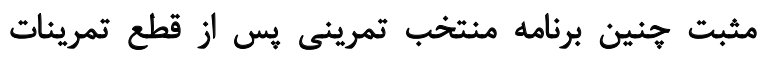
ويبريشن بررسى شود.

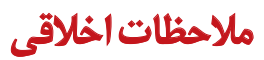

\section{ييروى أز اصول اخلاق يُووهش}

اين مقاله مورد تأييد كيمته اخلاق دانشكاه حكيم سبزوارى

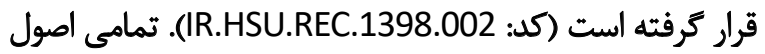

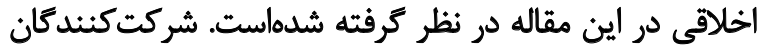

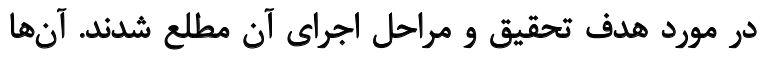

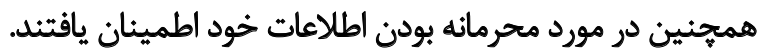

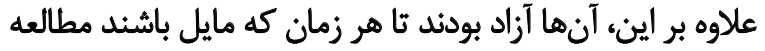

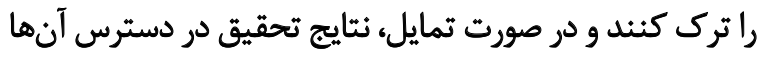

$$
\text { قرار تيرد. }
$$

$$
\text { حامى مالى }
$$

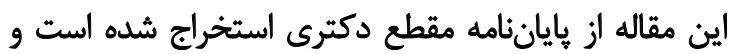

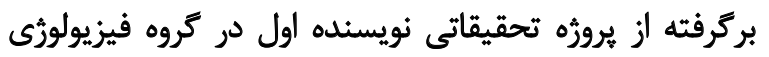
ورزشى، دانشكده علوم ورزشى، دانشكاه حكيم سبزوارى است.

$$
\text { مشاركت ثويسئدَّان }
$$

مفهوم سازى، نكارش - بررسى و ويرايش: همه نويسندكان.

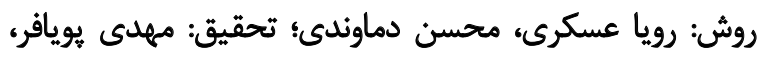

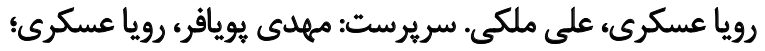
نظارت، تامين مالى: مهاكى يويافي مائر.

$$
\text { تعارض منافع }
$$

بنابر اظهار نويسندكان اين مقاله تعارض منافع ندارد. 


\section{References}

[1] Madureira MM, Takayama L, Gallinaro AL, Caparbo VF, Costa RA, Pereira RM. Balance training program is highly effective in improving functional status and reducing the risk of falls in elderly women with osteoporosis: A randomized controlled trial. Osteoporosis International. 2007; 18(4):419-25. [DOI:10.1007/s00198-006-0252-5] [PMID] [PMCID]

[2] Niewiadomski W, Cardinale M, Gasiorowska A, Cybulski G, Karuss B, Strasz A. Could vibration training be an alternative to resistance training in reversing sarcopenia? Journal of Human Kinetics. 2005; 14:3-20. http://www.johk.pl/ files/01niewiadomski.pdf

[3] Lopes K, Costa D, Santos L, Castro D, Bastone A. Prevalence of fear of falling among a population of older adults and its correlation with mobility, dynamic balance, risk and history of falls. Brazilian Journal of Physical Therapy. 2009; 13(3):223-9. [DOI:10.1590/S1413-35552009005000026]

[4] Gilasi HR, Soori H, Yazdani Sh, Taheri Tanjani P. [Fallrelated injuries in older people in Kashan (Persian)]. Journal of Paramedical Sciences and Rehabilitation. 2015; 4(3):74-82. [DOI:10.22038/JPSR.2015.4610]

[5] Nevitt MC, Cummings SR, Hudes ES. Risk factors for injurious falls: A prospective study. Journal of Gerontology.1991; 46(5):M164-70. [DOI:10.1093/geronj/46.5.M164] [PMID]

[6] Booth C E. Water exercise and its effect on balance and gait to reduce the risk of falling in older adults. Activities, Adaptation \& Aging. 2004; 28(4):45-57. [DOI:10.1300/J016v28n04_04]

[7] Delecluse C, Roelants M, Verschueren S. Strength increase after whole-body vibration compared with resistance training. Medicine \& Science in Sports \& Exercise. 2003; 35(6):1033-41. [DOI:10.1249/01.MSS.0000069752.96438.B0] [PMID]

[8] Fratini A, Cesarelli M, Bifulco P, LaGatta A, Pasquariello G. Analysis of muscle motion during whole body vibration training. Gait \& Posture. 2009; 30(Suppl 1):S67-8. [DOI:10.1016/j. gaitpost.2009.07.067]

[9] Rubin C, Recker R, Cullen D, Ryaby J, McCabe J, McLeod K. Prevention of postmenopausal bone loss by a low-magnitude, high-frequency mechanical stimuli: A clinical trial assessing compliance, efficacy and safety. Journal of Bone and Mineral Research. 2004; 19(3):343-51. [DOI:10.1359/JBMR.0301251] [PMID]

[10] Prisby RD, Lafage-Proust MH, Malaval L, Belli A, Vico L. Effects of whole body vibration on the skeleton and other organ systems in man and animal models: what we know and what we need to know. Ageing Research Reviews. 2008; 7(4):319-29. [DOI:10.1016/j.arr.2008.07.004] [PMID]

[11] Kiiski J, Heinonen A, Jaervinen TL, Kannus P, Sievanen H. Transmission of vertical whole body vibration to the human body. Journal of Bone and Mineral Research. 2008; 23(8):131825. [DOI:10.1359/jbmr.080315] [PMID]

[12] Brooke-Wavell K, Mansfield NJ. Risks and benefits of whole body vibration training in older people. Age and Ageing. 2009; 38(3): 254-5. [DOI:10.1093/ageing/afp036] [PMID]

[13] Bruyere O, Wuidart MA, Di Palma E, Gourlay M, Ethgen O, Richy F, et al. Controlled whole body vibration to decrease fall risk and improve health-related quality of life of nursing home residents. Archives of Physical Medicine and Rehabilitation. 2005; 86(2):303-7. [DOI:10.1016/j.apmr.2004.05.019] [PMID]
[14] Bogaerts A, Delecluse Ch, Boonen S, Claessens AL, Milisen $\mathrm{K}$, Verschueren SMP. Changes in balance, functional performance and fall risk following whole body vibration training and vitamin D supplementation in institutionalized elderly women. A 6 month randomized controlled trial. Gait \& Posture. 2011; 33(3):466-72. [DOI:10.1016/j.gaitpost.2010.12.027] [PMID]

[15] Chang SF, Lin PC, Yang RS, Yang RJ. The preliminary effect of whole-body vibration intervention on improving the skeletal muscle mass index, physical fitness, and quality of life among older people with sarcopenia. BMC Geriatrics. 2018; 18:17. [DOI:10.1186/s12877-018-0712-8] [PMID] [PMCID]

[16] Ghavi S, Golmohamadi B, Sohrabi M, Karimi N, Rahimi M, Sahaf R. [The effect of whole body vibration exercise, mental practice on balance of elderly men (Persian)]. Salmand: Iranian Journal of Ageing. 2015; 9(4):306-15. http://salmandj.uswr. ac.ir/article-1-770-fa.html

[17] Mikhael M, Orr R, Amsen F, Greene D, Singh MA. Effect of standing posture during whole body vibration training on muscle morphology and function in older adults: A randomised controlled trial. BMC Geriatrics. 2010; 10:74. [DOI:10.1186/1471-2318-10-74] [PMID] [PMCID]

[18] Rahimi M, Kordi M, Karimi N, Gaeini A, Samadi A, Alimoradi N. [The effects of whole body vibration training and creatine supplementation on lower extremity performance and balance in elderly males (Persian)]. Salmand: Iranian Journal of Ageing. 2011; 6(1):38-46. http://salmandj.uswr.ac.ir/ article-1-375-fa.html

[19] van Nes IJ, Latour H, Schils F, Meijer R, van Kuijk A, Geurts AC. Long-term effects of 6-week whole-body vibration on balance recovery and activities of daily living in the postacute phase of stroke: A randomized, controlled trial. Stroke. 2006; 37(9):2331-5. [DOI:10.1161/01.STR.0000236494.62957.f3] [PMID]

[20] Tseng SY, Hsu PS, Lai CL, Liao WC, Lee MC, Wang CH Effect of two frequencies of whole-body vibration training on balance and flexibility of the elderly: A randomized controlled trial. American Journal of Physical Medicine \& Rehabilitation. 2016; 95(10):730-7. [DOI:10.1097/PHM.0000000000000477] [PMID]

[21] Goodwin RD. Association between physical activity and mental disorders among adults in the United States. Preventive Medicine. 2003; 36(6):698-703. [DOI:10.1016/S00917435(03)00042-2]

[22] Stewart KJ. Exercise training: Can it improve cardiovascular health in patients with type 2 diabetes? British Journal of Sports Medicine. 2004; 38(3):250-2. [DOI:10.1016/S00917435(03)00042-2]

[23] Moran J, Ramirez-Campillo R, Granacher U. Effects of jumping exercise on muscular power in older adults: A meta analysis. Sports Medicine. 2018; 48(12):2843-57. [DOI:10.1007/ s40279-018-1002-5] [PMID]

[24] Lee B. Jump rope training. New York: Human Kinetics, 2010. https://www.google.com/books/edition/Jump_Rope Training/4rTinQEACAAJ?hl=en

[25] Coffey VG, Hawley JA. The molecular bases of training adaptation. Sports Medicine. 2007; 37(9):737-63. [DOI:10.2165/00007256-200737090-00001] [PMID]

[26] Miller RM, Heishman AD, Freitas EDS, Bemben MG. Comparing the acute effects of intermittent and continuous whole- 
body vibration exposure on neuromuscular and functional measures in sarcopenia and nonsarcopenic elderly women. Dose-Response. 2018; 16(3). [DOI:10.1177/1559325818797009] [PMID] [PMCID]

[27] Ko MC, Wu LS, Lee S, Wang CC, Lee PF, Tseng CY, et al. Whole-body vibration training improves balance control and sit-to-stand performance among middle-aged and older adults: A pilot randomized controlled trial. European Review of Aging and Physical Activity. 2017; 14:11. [DOI:10.1186/ s11556-017-0180-8] [PMID] [PMCID]

[28] Gotshalk LA, Kraemer WJ, Mendonca MA, Vingren JL, Kenny AM, Spiering BA, et al. Creatine supplementation improves muscular performance in older women. European Journal of Applied Physiology. 2008; 102(2):223-31. [DOI:10.1007/s00421-007-0580-y] [PMID]

[29] Jones CJ, Rikli RE. Measuring functional: Fitness of older adults. The Journal of Active Ageing. 2002; March April:24-30. https://www.dnbm.univr.it/documenti/OccorrenzaIns/ matdid/matdid182478.pdf

[30] Rikli RE, Jones CJ. Functional fitness normative scores for community-residing older adults, ages 60-94. Journal of Aging and Physical Activity. 1999; 7(2):162-81. [DOI:10.1123/ japa.7.2.162]

[31] Duncan PW, Weiner DK, Chandler J, Studenski S. Functional reach: A new clinical measure of balance. Journal of Gerontology. 1990; 45(6):M192-7. [DOI:10.1093/geronj/45.6.M192] [PMID]

[32] Beamis JF, Becker HD, Cavaliere S, Colt H, Diaz-Jimenez JP, Dumon JF, et al. ERS/ATS statement on interventional pulmonology. European Respiratory Journal. 2002; 19(2):356-73. [DO I:10.1183/09031936.02.00204602] [PMID]

[33] Liang X, Chen X, Li J, Yan M, Yang Y. Study on body composition and its correlation with obesity: A cohort study in 5121 Chinese Han participants. Medicine. 2018; 97(21):e10722. [DOI:10.1097/MD.0000000000010722] [PMID] [PMCID]

[34] Mofidi Sadr N, Askari R, Haghighi AH. [The effect of combined training (resistance - aerobic) on BMD and some of blood markers in obese and overweight postmenopausal women (Persian)]. Journal of Sabzevar University of Medical Sciences. 2019; 26(2):203-11. http://jsums.medsab.ac.ir/article_1173.html?lang=fa

[35] Karavatas SG, Tavakol K. Concurrent validity of Borg's rating of perceived exertion in African-American young adults, employing heart rate as the standard. The Internet Journal of Allied Health Sciences and Practice. 2005; 3(1):1-5. [DOI:10.46743/1540-580X/2005.1062]

[36] Álvarez-Barbosa F, del Pozo-Cruz J, del Pozo-Cruz B, Alfonso-Rosa RM, Rogers ME, Zhang Y. Effects of supervised whole body vibration exercise on fall risk factors, functional dependence and health-related quality of life in nursing home residents aged 80+. Maturitas. 2014; 79(4):456-63. [DOI:10.1016/j. maturitas.2014.09.010] [PMID]

[37] Milanese Ch, Piscitelli F, Zenti MG, Moghetti P, Sandri M, Zancanaro C. Ten-week whole-body vibration training improves body composition and muscle strength in obese women. International Journal of Medical Sciences. 2013; 10(3):30711. [DOI:10.7150/ijms.5161] [PMID] [PMCID]

[38] Maikala RV, King S, Bhambhani YN. Acute physiological responses in healthy men during whole-body vibration. Inter- national Archives of Occupational and Environmental Health. 2006; 79(2):103-14. [DOI:10.1007/s00420-005-0029-8] [PMID]

[39] Seif P, Dehkhoda MR, Rajabi H. [Effects of short term vibration training on some of physical fitness factors in elderly women (Persian)]. Research in Sport Medicine and Technology. 2011; 9(1):29-38. http://jsmt.khu.ac.ir/article-1-115-en. $\mathrm{html}$

[40] Corrie H, Brooke-Wavell K, Mansfield NJ, Cowley A, Morris R, Masud T. Effects of vertical and side-alternating vibration training on fall risk factors and bone turnover in older people at risk of falls. Age and Ageing. 2015; 44(1):115-22. [DOI:10.1093/ageing/afu136] [PMID]

[41] Burns P, Beekhuizen K, Jacobs P. Acute effects of wholebody vibration on lower body flexibility and strength. Medicine \& Science in Sports \& Exercise. 2004; 36(5):S350-1. [DOI:10.1249/00005768-200405001-01680]

[42] Rønnestad BR. Comparing the performance-enhancing effects of squats on a vibration platform with conventional squats in recreationally resistance-trained men. The Journal of Strength \& Conditioning Research. 2004; 18(4):839-45. [DOI:10.1519/00124278-200411000-00027] [PMID]

[43] Lim JH, Park CB, Kim BG. The effects of vibration foam roller applied to hamstring on the quadriceps electromyography activity and hamstring flexibility. Journal of Exercise Rehabilitation. 2019; 15(4):560-5. [DOI:10.12965/jer.1938238.119] [PMID] [PMCID]

[44] Goudarzian M, Ghavi S, Shariat A, Shirvani H, Rahimi M. Effects of whole body vibration training and mental training on mobility, neuromuscular performance, and muscle strength in older men. Journal of Exercise Rehabilitation. 2017; 13(5):573-80. [DOI:10.12965/jer.1735024.512] [PMID] [PMCID]

[45] Fagnani F, Giombini A, Di Cesare A, Pigozzi F, Di Salvo $\mathrm{V}$. The effects of a whole-body vibration program on muscle performance and flexibility in female athletes. American Journal o Physical Medicine \& Rehabilitation. 2006; 85(12):956-62. [DOI:10.1097/01.phm.0000247652.94486.92] [PMID]

[46] Allison SJ, Brooke-Wavell K, Folland J. High and odd impact exercise training improved physical function and fall risk factors in community-dwelling older men. Journal of Musculoskeletal \& Neuronal Interactions. 2018; 18(1):100-7. [PMID] [PMCID]

[47] Mester J, Spitzenpfeil P, Yue Z. Vibration loads: Potential for strength and power development. In: Komi PV, editor. Strength and Power in Sport. Oxford: Blackwell Science Ltd; 2003. pp. 488-501. [DOI:10.1002/9780470757215.ch24]

[48] Burke D, Hagbarth KE, Löfstedt L, Wallin BG. The responses of human muscle spindle endings to vibration during isometric contraction. The Journal of Physiology. 1976; 261(3):695711.[DOI:10.1113/jphysiol.1976.sp011581] [PMID] [PMCID]

[49] Karatrantou K, Bilios P, Bogdanis GC, Ioakimidis P, Soulas E, Gerodimos V. Effects of whole-body vibration training frequency on neuromuscular performance: A randomized controlled study. Biology of Sport. 2019; 36(3):273-82. [DOI:10.5114/biolsport.2019.87049] [PMID] [PMCID]

[50] Bokaeian HR, Bakhtiary AH, Mirmohammadkhani M, Moghimi J. The effect of adding whole body vibration training to strengthening training in the treatment of knee osteoarthritis: A randomized clinical trial. Journal of Bodywork 
and Movement Therapies. 2016; 20(2):334-40. [DOI:10.1016/j. jbmt.2015.08.005] [PMID]

[51] Broekmans T, Roelants M, Alders G, Feys P, Thijs H, Eijnde BO. Exploring the effects of a 20-week whole-body vibration training programme on leg muscle performance and function in persons with multiple sclerosis. Journal of Rehabilitation Medicine. 2010; 42(9):866-72. [DOI:10.2340/16501977-0609] [PMID]

[52] Arabasadi M, Kordi MR, Gaeini AA. [The effect of vibration training on skill related fitness of trained and untrained student girls (Persian)]. Journal of Movement Science \& Sports. 2010; 8(15):13-23. https://www.sid.ir/fa/journal/ViewPaper.aspx?ID=159645

[53] Wang P, Yang L, Liu C, Wei X, Yang X, Zhou Y, et al. Effects of whole body vibration exercise associated with quadriceps resistance exercise on functioning and quality of life in patients with knee osteoarthritis: A randomized controlled trial. Clinical Rehabilitation. 2016; 30(11):1074-87. [DOI:10.1177/0269215515607970] [PMID]

[54] Spielmanns M, Gloeckl R, Gropp JM, Nell Ch, Koczulla AR, Boeselt $\mathrm{T}$, et al. Whole-body vibration training during a low frequency outpatient exercise training program in chronic obstructive pulmonary disease patients: A randomized, controlled trial. Journal of Clinical Medicine Research. 2017; 9(5):396-402. [DOI:10.14740/jocmr2763w] [PMID] [PMCID]

[55] Chen CC, Lin YC. Jumping rope intervention on health-related physical fitness in students with intellectual impairment. The Journal of Human Resource and Adult Learning. 2012; 8(1):56-62. http://www.hraljournal.com/Page/6\%20Chen, \%20 Chao-Chien.pdf

[56] Wei N, Pang MY, Ng SS, Ng GY. Optimal frequency/ time combination of whole body vibration training for developing physical performance of people with sarcopenia: A randomized controlled trial. Clinical Rehabilitation. 2017; 31(10):1313-21. [DOI:10.1177/0269215517698835] [PMID]

[57] Verschueren SMP, Roelants M, Delecluse Ch, Swinnen S, Vanderschueren D, Boonen S. Effect of 6-month whole body vibration training on hip density, muscle strength, and postural control in postmenopausal women: A randomized controlled pilot study. Journal of Bone and Mineral Research. 2004; 19(3):352-9. [DOI:10.1359/JBMR.0301245] [PMID] 
This Page Intentionally Left Blank 